\title{
Mass independent sulfur isotope signatures in CMs: implications for sulfur chemistry in the early
} solar system

\author{
J. Labidia, J. Farquharb, C.M.O’'D. Alexanderc, D.L. Eldridgeb, H. Oduro ${ }^{d}$
}

\begin{abstract}
Affiliations:
${ }^{a}$ Geophysical Laboratory, Carnegie Institution of Washington, Washington, D.C. 20015, USA.

${ }^{\mathrm{b}}$ Department of Geology, University of Maryland, College Park MD, 20740, USA

${ }^{c}$ Department of Terrestrial Magnetism, Carnegie Institution of Washington, Washington, D.C. 20015, USA.

${ }^{\mathrm{d} D e p a r t m e n t ~ o f ~ E a r t h ~ a n d ~ E n v i r o n m e n t a l ~ S c i e n c e s, ~ S t ~ A n d r e w s ~ U n i v e r s i t y, ~ F i f e ~ K Y 16 ~ 9 A J, ~ U n i t e d ~}$

Kingdom

*Correspondence to: jabrane.labidi@uni-tuebingen.de
\end{abstract}

Keywords: sulfur isotope, photodissociation, early solar system, chondrites 


\section{Abstract}

We have investigated the quadruple sulfur isotopic composition of inorganic sulfur-bearing phases from 13 carbonaceous chondrites of CM type. Our samples include 4 falls and 9 Antarctic finds. We extracted sulfur from sulfides, sulfates, and elemental sulfur $\left(\mathrm{S}^{0}\right)$ from all samples. On average, we recover a bulk

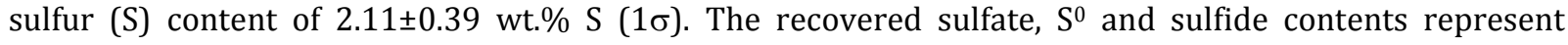
$25 \pm 12 \%, 10 \pm 7 \%$ and $65 \pm 15 \%$ of the bulk S, respectively (all $1 \sigma$ ). There is no evidence for differences in the bulk S content between falls and finds, and there is no correlation between the S speciation and the extent of aqueous alteration. We report ranges of $\Delta^{33} S$ and $\Delta^{36} S$ values in CMs that are significantly larger than previously observed. The largest variations are exhibited by $S^{0}$, with $\Delta^{33} S$ values ranging between $0.104 \pm 0.012 \%$ and $+0.256 \pm 0.018 \%$ o $(2 \sigma)$. The $\Delta^{36} \mathrm{~S} / \Delta^{33} \mathrm{~S}$ ratios of $\mathrm{S}^{0}$ are on average $-3.1 \pm 1.0(2 \sigma)$. Two CMs show distinct $\Delta^{36} \mathrm{~S} / \Delta^{33} \mathrm{~S}$ ratios, of $+1.3 \pm 0.1$ and $+0.9 \pm 0.1$. We suggest that these mass independent $\mathrm{S}$ isotopic compositions record $\mathrm{H}_{2} \mathrm{~S}$ photodissociation in the nebula. The varying $\Delta^{36} \mathrm{~S} / \Delta^{33} \mathrm{~S}$ ratios are interpreted to reflect photodissociation that occurred at different UV wavelengths. The preservation of these isotopic features requires that the S-bearing phases were heterogeneously accreted to the CM parent body. Nonzero $\Delta^{33} S$ values are also preserved in sulfide and sulfate, and are positively correlated with $\mathrm{S}^{0}$ values. This indicates a genetic relationship between the S-bearing phases: We argue that sulfates were produced by the direct oxidation of $S^{0}$ (not sulfide) in the parent body. We describe two types of models that, although imperfect, can explain the major features of the CM S isotope compositions, and can be tested in future studies. Sulfide and $\mathrm{S}^{0}$ could both be condensates from the nebula, as the residue and product, respectively, of incomplete $\mathrm{H}_{2} \mathrm{~S}$ photodissociation by UV light (wavelength $<150 \mathrm{~nm}$ ). This idea requires that $\mathrm{FeS}$ formation and the $\mathrm{S}^{0}$ condensation co-occur. As an alternative, ice accretion to the CM parent body could allow the delivery of S-MIF in CMs. In that case, sulfides would have been the only S-bearing condensate in CM precursors, and $\mathrm{S}^{0}$ would have been derived from the oxidation of $\mathrm{H}_{2} \mathrm{~S}$ trapped in ices, after its photodissociation at low temperature $(<500 \mathrm{~K})$ in the nebula. In our models, the observations of $\mathrm{H}_{2} \mathrm{~S} U \mathrm{VV}$ photodissociation is required to occur at the disk surface, and allowed in nebular environments with canonical C/O ratios. Vertical motions in the disk would redistribute phases that condensed at high altitude to the midplane, where they accreted in the phases that make up the chondritic matrix. 


\section{Introduction}

The sulfur (S) isotopic compositions of meteorites reflect a complex collection of nucleosynthetic, physical, and chemical processes that occurred both in nebular and asteroidal environments. The magnitude of mass-independent $\mathrm{S}$ isotope signatures in meteorites is typically sub-permil, nowhere near as large as those seen for oxygen. However, a growing body of evidence suggests that they are widespread, and these signatures have largely been interpreted to reflect photochemical reactions (Cooper et al., 1997, Farquhar et al., 2000a; Rai et al., 2005; Chakraborty et al., 2013; Antonelli et al., 2014).

Subtle but widespread $\Delta^{33} S$ variations have been reported in achondrites and iron meteorites, with $\Delta^{33} \mathrm{~S}$ variations extending up to $0.050 \%$ for bulk S (Farquhar et al. 2000a, Rai et al. 2005, Antonelli et al. 2014, DeFouilloy et al. 2016). Aubrites carry only small ${ }^{33}$ S enrichments relative to Canyon Diablo Troilite (CDT) for their bulk S, with a mean $\Delta^{33}$ S value of $+0.015 \pm 0.006 \%$ o $(n=6$, Rai et al., $2005,2 \sigma$ ), but an oldhamite (CaS) mineral separate from the Norton County aubrite was reported to have a $\Delta^{33} S$ value of $+0.161 \pm 0.012 \%$ o $(2 \sigma$, Rai et al., 2005). DeFouilloy et al. (2016) reported near zero to significantly negative $\Delta^{33} \mathrm{~S}$ values in oldhamites ( $\left.\mathrm{CaS}\right)$ from aubrites, down to $-0.085 \pm 0.020 \%$ o $(2 \sigma)$ for Norton County. Because the Rai et al. (2005) result constitutes the highest $\Delta^{33} S$ value in the meteorite inorganic $S$ record, oldhamite was suggested as a carrier for S-MIF in aubrites and other meteorites (Rai et al. 2005). Oldhamite being a relatively refractory mineral would have acquired its S-MIF signatures in the inner, hotter and reducing parts of the nebula (Rai et al., 2005). After condensation, a heterogeneous distribution of CaS grains in the precursors of achondrites could account for their variable $\Delta^{33}$ S values (Rai et al., 2005). Antonelli et al. (2014) also suggested that refractory sulfides, such as oldhamite, would have acted as carriers of S-MIF to iron meteorite precursors.

In contrast to the achondrites, the chondrites have been considered to preserve evidence for predominantly mass dependent sulfur. Gao and Thiemens (1993a) reported mass dependent S isotope ratios in seven carbonaceous chondrites, three of which were CM chondrites (Mighei, Allan Hills (ALH) 84029, and Murchison), and observations of S-MIF signature have been considered to be isolated occurrences. One of these observations includes S-MIF identified in a minor component of the CV chondrite 
Allende (Rees and Thode, 1977: $\Delta^{33} S=+1.1 \pm 0.1 \%$ 2 $\sigma$ ). A second observation is for the sulfonic organic acids from the Murchison CM chondrite that carry $\Delta^{33}$ S values between $-0.40 \pm 0.05 \%$ and $+2.00 \pm 0.05 \%$ o (Cooper et al., 1997, 2 $\sigma$ ). Smaller magnitude S-MIFs were also observed in chondrules of the Djahala ordinary chondrite (Rai and Thiemens, 2007: $\Delta^{33} \mathrm{~S}=+0.10 \pm 0.03 \%$, $2 \sigma$ ). Rees and Thode (1977) considered their observation as a possible nucleosynthetic anomaly, whereas Cooper et al., (1997) suggested that the organic acids S isotopic compositions reflected the composition of photo-processed interstellar S. Rai and Thiemens (2007) envisioned a very different scenario that tied anomalies in chondrules to those in achondrites. The X-wind astrophysical model (Shu et al., 1997) was suggested as a way to redistribute CaS grains from a region close to the Sun where they formed (and captured S-MIF) to other parts of the early solar nebula where the precursors to achondrites formed (Rai et al. 2005). This model was favoured to account for the $\Delta^{33} \mathrm{~S}$ value in sulfides extracted from Djahala $(\mathrm{H} 3.8)$ chondrules $(+0.10 \pm 0.03 \%, 2 \sigma)$, because it provides a "mechanism for chondrule precursors to be irradiated with high energy UV light from the early Sun" (Rai and Thiemens, 2007).

The X-wind model is, however, hard to reconcile with several major features of chondrites. For example, it predicts highly oxidizing formation conditions for CAIs in the X-region, due to the removal of $\mathrm{H}_{2}$ (Shu et al., 2001). However, oxygen barometers of CAI formation indicate a gas of solar composition (Simon et al., 2007). In addition, the $\mathrm{X}$-wind model does not allow chondrules to form from material containing primary $\mathrm{S}$, as the starting temperatures experienced by the precursors of chondrules would be too high $(\sim 1100 \mathrm{~K}$ in Shu et al., 2001, which is $\sim 400 \mathrm{~K}$ above the FeS condensation temperature). Primary and secondary Fe sulfides are, however, common phases in chondrules (i.e., Tachibana and Huss, 2005; Marrocchi and Libourel, 2013). For more details, the reader is referred to review contributions (Krot et al., 2009; Desch et al., 2010).

Carbonaceous chondrites of CM type are often considered as among the most primitive chondrites (e.g., Huss et al., 2003), although they have clearly experienced varying degree of aqueous alteration (e.g., Rubin et al., 2007; Alexander et al., 2013; Howard et al., 2015 and references therein). The CMs have amongst the highest bulk S contents of any chondrite group (Dreibus et al., 1995; Burgess et al., 1991), and host various S-bearing phases, including $\mathrm{Ni}$-Fe sulfides and tochilinite, elemental $\mathrm{S}\left(\mathrm{S}^{0}\right)$, and sulfates (Airieau et al. 2005, 
108 Bullock et al. 2010, Gao and Thiemens, 1993, Rubin et al. 2007). These meteorites may thus have recorded

109 and preserved the complex S chemistry that occurred in the nebula and during parent body processes.

110 Here, we report S isotope data for $13 \mathrm{CM}$ carbonaceous chondrites that preserve anomalous $\Delta^{33 \mathrm{~S}}$ values.

111 We show that a photolytic origin in the hotter, inner parts of the solar nebula, combined with a mixing

112 process such as that provided by the X-wind does not explain the occurrence of S-MIF in CMs, and may not

113 be required to account for S-MIF in achondrite parent bodies. Our data support the idea that S-MIF was produced by $\mathrm{H}_{2} \mathrm{~S}$ photolysis under low temperature conditions near the surface of the disk, and imply that

115 S-MIF can potentially be produced at various heliocentric distances. We discuss whether it could constitute

116 a viable alternative to the idea of a refractory high-temperature sulfide condensate such as oldhamite as

117 the origin of the signature in the parent bodies of achondrites and iron meteorites as well as in chondrules.

\section{Samples}

120

Thirteen meteorites were studied here, including four observed falls and nine Antarctic finds (Table 1).

121 Samples were chips from the interiors of the meteorites taken well away from the fusion crust to minimize

122 the effects of terrestrial contamination and modification during atmospheric entry. Other than the

123 Antarctic samples allocated by the Johnson Space Center that were stored under $\mathrm{N}_{2}$ atmosphere for

124 curation and subsequently in desiccators, no special precautions were taken with regard to sample storage

125 history. The samples were selected to cover the full range of CM aqueous alteration (Table 1). These CMs

126 were previously divided into unheated and heated meteorites based on their water contents and hydrogen

127 isotopic composition (Alexander et al., 2013). Here, 12 unheated CMs and one heated CM were 128 investigated.

129 Samples of meteorite falls include Murchison and Cold Bokkeveld (allocated by the British Natural 130 History Museum), as well as Banten and Nogoya (allocated by the Smithsonian Institution). With the 131 exception of Murchison and Cold Bokkeveld, all the processed powders were previously investigated for 132 their bulk hydrogen, nitrogen and carbon isotope compositions (Alexander et al., 2012, 2013). The H, N and 133 C isotope compositions of their insoluble organic matter (IOM) (Alexander et al., 2007, 2010), as well as 134 their carbonate $\mathrm{C}$ and $\mathrm{O}$ isotope composition (Alexander et al., 2015) have also been reported for most of 135 the samples. The $\mathrm{H}$ contents and isotope compositions (Alexander et al., 2013) and bulk mineralogies 
136 (Howard et al., 2015) of the samples have been used to place the samples on an alteration sequence. 137 Murchison and Cold Bokkeveld were also investigated by Alexander et al. $(2007,2012,2013,2015)$, but on 138 different samples.

139 The samples were crushed in a steel percussion mortar and then ground in an agate mortar to pass 140 through 100-150 $\mu \mathrm{m}$ sieves. About $200 \mathrm{mg}$ aliquots were taken from the $\sim 1000 \mathrm{mg}$ powdered samples that 141 had been previously studied by Alexander et al. $(2012,2013,2015)$.

\section{Methods}

144

\subsection{Extraction}

Various authors have reported the occurrence of $\mathrm{S}^{0}$ in CIs and CMs, in addition to ubiquitous sulfides. Monster et al. (1965), Kaplan and Thode (1966) and Gao and Thiemens (1993) used wet chemistry techniques to recover $S^{0}$. The main strategy these studies employed was to process the samples in organic solvents to selectively dissolve $\mathrm{S}^{0}$. Burgess et al. (1991) used a stepped combustion technique to survey the relative and absolute abundances of $\mathrm{S}^{0}$ in various carbonaceous chondrites (CI, CM and CR). Sulfates have also been previously observed in carbonaceous chondrites, especially in CMs. Fuchs et al. (1973) observed gypsum plates in both Murchison and Cold Bokkeveld. Although petrographic studies of CMs report sulfides to be the dominant (if not the exclusive) observed S-bearing phase (e.g., Bullock et al., 2005, 2010), Gao and Thiemens (1993) report sulfate as being the dominant S-bearing pool in CMs. Hence, we developed an extraction protocol in anticipation of the complex S speciation in CMs. We present here a technique for redox-specific S extraction out of CMs that allows for determination of the S speciation, abundances and isotopic compositions for three recovered S-pools: $\mathrm{S}^{0}$, sulfate, and sulfide.

We used sequential extraction techniques to extract $S$ from these three pools. Organic $S$ was not the target of this study, and will not be further discussed. The protocol is summarized in Fig. 1 and is described below. Samples were first processed in $\mathrm{N}_{2}$-flushed Milli-Q water for 24 hours at room temperature to 160 dissolve sulfate and soluble organic compounds. After centrifugation and extraction of the water, we added $161 \mathrm{BaCl}_{2}$ to the water solutions leading to the precipitation of $\mathrm{BaSO}_{4}$ from dissolved sulfate. This step yielded the equivalent of from $1100 \pm 100$ ppm S (MacKay Glacier (MCY) 05230) to $12900 \pm 100$ ppm S (Pecora 163 Escarpment (PCA) 91084). For 10 of the 13 samples, the sulfate contents were more tightly constrained to 
164 4500-6000 ppm S. After rinsing with Milli-Q water, this extraction was repeated and yielded non165 detectable $(<50 \mathrm{ppm})$ sulfate in the repeat, indicating that the first extraction was quantitative. It also 166 indicates that our protocol does not lead to sulfate formation via sulfide oxidation, as it would otherwise 167 yield significant $\mathrm{SO}_{4}{ }^{2-}$ amounts in the repeat as well. The $\mathrm{S}$ in our $\mathrm{BaSO}_{4}$ precipitates was then reduced to $168 \mathrm{H}_{2} \mathrm{~S}$ and recovered as silver sulfide $\left(\mathrm{Ag}_{2} \mathrm{~S}\right)$ using the standard technique described by Thode et al. (1961).

169 Elemental sulfur is insoluble in water at room temperature $(19 \pm 6 \mathrm{nM}$; Boulegue, 1978). After rinsing,

170 the rock samples were processed in $\mathrm{N}_{2}$-flushed ethanol (reagent grade, anhydrous 200 proof) for 72 hours

171 at room temperature to extract $S^{0}$. The ethanol solutions then underwent reaction with a sub-boiling

$172\left(\sim 80^{\circ} \mathrm{C}\right) 5 \mathrm{~N} \mathrm{HCl}$ solution for 1 hour. At this step, any hypothetical ethanol-soluble, acid-volatile sulfide 173 phases would be converted into $\mathrm{H}_{2} \mathrm{~S}$ and recovered as $\mathrm{Ag}_{2} \mathrm{~S}$. This step, however, only yielded blank level 174 amounts of $S$ for all samples $(<1 \mathrm{ppm} \mathrm{S})$, ruling out the occurrence of any such phases. The solutions were 175 then processed in sub-boiling $\mathrm{CrCl}_{2}+\mathrm{HCl}$ solutions for 3 hours using the standard technique described by 176 Canfield et al. (1986), which quantitatively reduces $\mathrm{S}^{0}$ to $\mathrm{H}_{2} \mathrm{~S}$ via the oxidation of $\mathrm{Cr}^{2+}$ to $\mathrm{Cr}^{3+}$ and allows for 177 the recovery of $\mathrm{H}_{2} \mathrm{~S}$ as $\mathrm{Ag}_{2} \mathrm{~S}$. Between $400 \pm 100 \mathrm{ppm}$ and $5500 \pm 100 \mathrm{ppm} \mathrm{S}$ was extracted during this step, 178 with most samples yielding $<2500 \mathrm{ppm} \mathrm{S.} \mathrm{Aliquots} \mathrm{of} \mathrm{these} \mathrm{ethanol} \mathrm{extracts} \mathrm{were} \mathrm{also} \mathrm{processed} \mathrm{for}$ 179 independent $\mathrm{S}^{0}$ analysis via High Performance Liquid Chromatography (HPLC) with UV-detection 180 (Kamyshny et al., 2009). An Agilent 1200 HPLC system with an Agilent Eclipse XDB-C18 column (4.6 x 150 $181 \mathrm{~mm} ; 5 \mu \mathrm{m}$ particle size) was utilized with an isocratic 100\% HPLC-grade methanol mobile phase. 182 Calibrations for the quantification of $\mathrm{S}^{0}$ were prepared from stock solutions of $\mathrm{S}^{0}$ (reagent grade refined $\mathrm{S}$ 183 powder, 100 mesh; Sigma Aldrich) dissolved in ethanol (reagent grade, anhydrous 200 proof) over a 184 concentration range of $1-1000 \mu \mathrm{M}$ (retention times of ca. 5.5 min with our method). The HPLC 185 determinations of $\mathrm{S}^{0}$ matched our wet chemistry extractions across the large range of observed $\mathrm{S}$ 186 abundances within $\pm 10 \%$ (supplementary Fig. 1 and Table 2). The convergence of these two independent 187 approaches strongly indicates that the S extracted with ethanol is predominately in the form of $\mathrm{S}^{0}$. We 188 further argue below that this S-bearing pool is naturally occurring $\mathrm{S}^{0}$ in the CMs. As for the water 189 extractions, this step was repeated and did not yield any significant additional S extraction (i.e., always < $1902 \%$ of what was extracted in the first step), indicating that the first extraction was quantitative. It also 
191 indicates that the protocol did not lead to the formation of $S^{0}$ from sulfide oxidation, as it would otherwise 192 lead to significant $\mathrm{S}^{0}$ amounts in the repeat.

193 After rinsing with Milli-Q water, the rock samples were digested in a sub-boiling $5 \mathrm{~N} \mathrm{HCl}$ solution for 3 194 hours. $\mathrm{H}_{2} \mathrm{~S}$ was evolved from all samples (recovered as $\mathrm{Ag}_{2} \mathrm{~S}$ ) and is referred to as the acid-volatile $\mathrm{S}$ (AVS) 195 fraction. Such fractions are usually considered to be the products of monosulfide digestion (e.g., FeS, NiS, 196 but not pyrite, see Canfield et al., 1986). Between 100 and 3100 ppm S were extracted at this step. For 197 three samples, AVS extractions yielded $100 \mathrm{ppm}$, which was insufficient to analyze their isotopic 198 compositions.

199 Solid residues and acid solutions from the $\mathrm{HCl}$ treatments were then transferred to Falcon ${ }^{\mathrm{TM}}$ tubes for 200 centrifugation. The acid solution was subsequently transferred back to the distillation apparatus, and 201 underwent reaction with a sub-boiling $\mathrm{CrCl}_{2}$ solution (after Canfield et al., 1986). $\mathrm{H}_{2} \mathrm{~S}$ was evolved for all 202 samples and recovered as $\mathrm{Ag}_{2} \mathrm{~S}$. This fraction is referred to as step 12. Between $200 \mathrm{ppm} \mathrm{S}$ and $1000 \mathrm{ppm} \mathrm{S}$ 203 were extracted at this step. The solid residue of the AVS extraction was processed in $\mathrm{N}_{2}$-flushed ethanol 204 (reagent grade, anhydrous 200 proof) for 72 hours at room temperature. The ethanol extracts were then 205 processed in a sub-boiling $5 \mathrm{~N} \mathrm{HCl}$ solution for 1 hour. As for the first ethanol extraction steps (step 2), this never yielded detectable AVS, ruling out the occurrence of any acid volatile sulfide phases in the ethanol solutions. The solutions were then processed in sub-boiling $\mathrm{CrCl}_{2}$ solution for 3 hours. Again, evolved $\mathrm{H}_{2} \mathrm{~S}$ was recovered as $\mathrm{Ag}_{2} \mathrm{~S}$. Between $5400 \mathrm{ppm}$ and $19000 \mathrm{ppm} \mathrm{S}$ was extracted, which represent roughly an order of magnitude more than in step 12. This fraction is referred to as step 13. The AVS, step 12 and step

21013 extracts were analyzed separately for their S isotopic compositions, and the results are given in Table 2.

211 These three extractions (AVS, step 12 and step 13) were pooled by mass balance and considered to 212 represent the bulk sulfide (see below).

\subsection{Isotopic Analysis}

216 fluorination with at least 10 times excess of pure $\mathrm{F}_{2}$ at $250{ }^{\circ} \mathrm{C}$ overnight. The produced $\mathrm{SF}_{6}$ was isolated 217 from impurities with cryogenic and chromatographic techniques, as described in Bains-Sahota and 218 Thiemens (1988), Rumble et al. (1993) and modified by Ono et al. (2006). The volume of purified SF 6 was 
219 then measured with a manometer, and transferred to the dual inlet of a ThermoFinnigan MAT 253 isotope ratio gas-source mass spectrometer that was used to measure ion beams at $\mathrm{m} / \mathrm{z}=127+, 128+, 129+$ and

$221131+$. Once $\delta^{n} S$ are determined $(n=33,34,36), \Delta^{33} S$ and $\Delta^{36} S$ are calculated $\left(\Delta^{33} S=\delta^{33} S-1000\left(\left(\delta^{34} S / 1000\right.\right.\right.$ $\left.222+1)^{0.515}-1\right)$ and $\left.\left.\Delta^{36} S=\delta^{36} S-1000\left(\left(\delta^{34} S / 1000+1\right)^{1.90}-1\right)\right)\right)$. The results are given in Table 3. IAEA S1 223 values are used to evaluate our long-term accuracy and precision on standards, and to anchor our $\delta^{34} S$ to 224 the V-CDT scale. Analyses of the S1 standard performed along with the CM analyses yield average $\delta^{34} \mathrm{~S}, \Delta^{33} \mathrm{~S}$ and $\Delta^{36}$ S values of $-5.28 \pm 0.09 \%,+0.083 \pm 0.008 \%$ and $-0.78 \pm 0.26 \%$ o $(n=13$, all 2s.d.) relative to our reference gas. Relative to the same reference gas, our CDT yield $\delta^{34} \mathrm{~S}, \Delta^{33} \mathrm{~S}$ and $\Delta^{36} \mathrm{~S}$ values at $-4.88 \pm 0.15 \%$, $-0.035 \pm 0.008 \%$ and $-0.08 \pm 0.08 \%$ ( $n=6$, all 2 s.d.). The $S$ isotope shift for $\delta^{34} S, \Delta^{33} \mathrm{~S}$ and $\Delta^{36} \mathrm{~S}$ between CDT and S1 is comparable to what was obtained elsewhere (Ono et al., 2006; Labidi et al., 2012).

We use the CDT scale to anchor our $\delta^{34} S, \Delta^{33} S$ and $\Delta^{36} S$ values. We additionally report our $\delta^{34} S$ values on the V-CDT scale to allow comparisons with other datasets also reported relative to V-CDT (e.g., terrestrial mantle-derived rocks, Labidi et al. 2013). We obtained $2 \sigma$ uncertainties of $0.10,0.012$ and $0.25 \%$ for $\delta^{34}$, $\Delta^{33} \mathrm{~S}$ and $\Delta^{36} \mathrm{~S}$ values, respectively, for all measurements with a $\mathrm{SF}_{6}$ amount $>1 \mu$ mol. When the $\mathrm{SF}_{6}$ amount was $<1 \mu \mathrm{mol}(\mathrm{n}=4)$, we obtained $2 \sigma$ uncertainties of $0.10,0.018$ and $0.35 \%$ for $\delta^{34} \mathrm{~S}, \Delta^{33} \mathrm{~S}$ and $\Delta^{36} \mathrm{~S}$ values, respectively (Table 3). The whole procedure of S extraction and isotope analysis was repeated once on Murchison, and twice on Cold Bokkeveld, to address sample heterogeneity at the scale of the studied 236 powders (Table 4).

\section{Results}

\subsection{Bulk $S$ content}

The $\mathrm{S}$ abundances in the five extracted $\mathrm{S}$ reservoirs of each sample are reported in Table 1, and shown

241 in Fig. 2. Bulk S contents range between 1.72 \pm 0.2 wt.\% S (Graves Nunataks (GRA) 98074) and 3.01 \pm 0.2

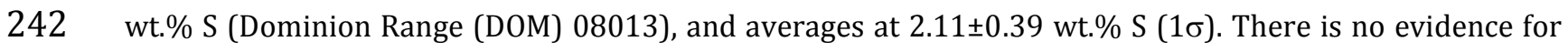
243 differences in the bulk S content between falls and finds. We find Antarctic finds to have an average bulk S content of $2.18 \pm 0.43 \mathrm{wt} . \%(\mathrm{n}=9,1 \sigma)$, whereas the finds have an average of $1.95 \pm 0.29 \mathrm{wt} . \%(n=4,1 \sigma)$. 


\subsection{Sulfur speciation}

For all the studied CMs, three S pools were systematically recovered and analyzed: Sulfide, sulfate, and

$248 \mathrm{~S}^{0}$. The absolute abundances of each pool are given in Table 2 and shown in Fig. 2. The average sulfate 249 content of our samples is $5400 \pm 3000 \mathrm{ppm} \mathrm{S} \mathrm{(n=13),} \mathrm{but} \mathrm{the} \mathrm{contents} \mathrm{range} \mathrm{between} 1100 \pm 100 \mathrm{ppm} \mathrm{S}$ 250 (MCY 05230) to $12900 \pm 100$ ppm S (PCA 91084). Exclusion of these two extreme samples significantly 251 decreases the standard deviation, leading to an average value of $5100 \pm 1700 \mathrm{ppm} \mathrm{S}(\mathrm{n}=11,1 \sigma)$. Fall and find sulfate contents average at $5400 \pm 1200 \mathrm{ppm} \mathrm{S}(\mathrm{n}=4,1 \sigma)$ and $5400 \pm 3500 \mathrm{ppm} \mathrm{S}(\mathrm{n}=9,1 \sigma)$, respectively. The recovered sulfate contents represent $25 \pm 12 \%$ of the bulk $S$ of the studied CM chondrites $(n=13,1 \sigma, 6 \%$ for MCY 05230, 47\% for PCA 91084).

Elemental S abundances recovered from the ethanol extractions average at $1900 \pm 1400$ ppm S, and range between $400 \pm 100$ ppm S (Lewis Cliffs (LEW) 85312) and 5500 \pm 100 ppm S (Allan Hills (ALH) 84029). Fall and find $S^{0}$ contents average at $1300 \pm 300 \mathrm{ppm} \mathrm{S}(\mathrm{n}=4,1 \sigma)$ and $2222 \pm 1600 \mathrm{ppm} \mathrm{S}(\mathrm{n}=9,1 \sigma)$, 84029).

260 Sulfides are the dominant pool in every chondrite studied here, with the exception of PCA 91084.

261 Sulfide contents average at $13700 \pm 3600 \mathrm{ppm} \mathrm{S}$, and ranges between $9500 \pm 100 \mathrm{ppm}$ S (ALH 84029) and $26220800 \pm 100$ ppm S (DOM 08013). These values represent $65 \pm 15 \%$ of the bulk $S(n=13,1 \sigma, 48 \%$ for ALH 94029, 69\% for DOM 08013). Note that PCA 91084 is the only CM where sulfide is not the dominant pool sulfide and sulfate represent $41 \%$ and $47 \%$ of its bulk S, respectively. Fall and find sulfide contents average at $12900 \pm 3900 \mathrm{ppm} \mathrm{S}(\mathrm{n}=4,1 \sigma)$ and $14100 \pm 3700 \mathrm{ppm} \mathrm{S}(\mathrm{n}=9,1 \sigma)$, respectively.

\subsection{Bulk $S$ isotope compositions}

The average bulk $\mathrm{S} \delta^{34} \mathrm{~S}$ for all CMs is $-0.08 \pm 0.44 \% 0(1 \sigma, \mathrm{n}=13)$ versus our CDT estimate. The average $\Delta^{33} \mathrm{~S}$ and $\Delta^{36} \mathrm{~S}$ are $+0.021 \pm 0.071 \%$ and $0.00 \pm 0.09 \%$, respectively $(1 \sigma, \mathrm{n}=13)$, relative to CDT. The standard 
273 (LEW 85312) and $+0.207 \pm 0.008 \%$ o (Murchison) relative to CDT (Fig. 3). Bulk S $\Delta^{36}$ S values range from $2740.11 \pm 0.25 \%$ o (PCA 91084) and $+0.22 \pm 0.25 \%$ (MCY 05230) relative to CDT (Fig. 3b). Although the two CM 275 with significantly positive $\Delta^{33} \mathrm{~S}$ both display positive $\delta^{34} \mathrm{~S}$, there is no systematic trend between $\delta^{34} \mathrm{~S}$ 276 and $\Delta^{33}$ S. $\Delta^{36}$ S values remain within uncertainties indistinguishable from CDT, and no trends appear in a $277 \Delta^{33} \mathrm{~S}$ 回回回 $\Delta^{36} \mathrm{~S}$ plot. Additionally, no systematic S isotope difference was observed between falls and finds.

\section{4 $\mathrm{S}$ isotopic compositions of sulfide, sulfate and elemental sulfur}

The $\mathrm{S}$ isotope compositions of the sulfide pools in the meteorites were estimated by combining the AVS,

step 12 and step 13 results (section 5.1 .3$)$. The calculated CM average sulfide $\delta^{34} \mathrm{~S}$ is $-0.15 \pm 0.33 \%$ o $(1 \sigma$, 282 $n=13$ ) relative to CDT, with values for individual meteorites ranging between $-0.70 \pm 0.15 \%$ (DOM 08003 ) and $+0.18 \pm 0.15 \%$ (MCY 05230$)$. The average CM sulfide $\Delta^{33} \mathrm{~S}$ is $+0.015 \pm 0.061 \% 0(1 \sigma, \mathrm{n}=13)$, with values ranging between $-0.070 \pm 0.008 \%$ (LEW 85312 ) and $+0.177 \pm 0.008 \%$ (split 2, Murchison - table 4 ). For $\Delta^{36} \mathrm{~S}$, average $\mathrm{CM}$ value is $+0.04 \pm 0.11 \%$ o $(1 \sigma, \mathrm{n}=13$ ), with values ranging between $-0.10 \pm 0.09 \%$ (LEW 87022 ) and $+0.22 \pm 0.23 \%$ (DOM 08003). The variability in the sulfide $\mathrm{S}$ isotopic compositions is comparable to the variability observed for bulk $\mathrm{S}$, as illustrated by the comparable (or lower) standard deviations of the average values (Table 3).

Sulfate and $\mathrm{S}^{0}$ have $\mathrm{S}$ isotope compositions that are more variable than those of the sulfide. The average

$\mathrm{CM} \delta^{34} \mathrm{~S}$ for sulfate is $-1.15 \pm 1.48 \%$ o $(1 \sigma, \mathrm{n}=13)$ relative to $\mathrm{CDT}$, with values ranging over $\sim 6 \%$ between $4.54 \pm 0.50 \%$ o (LEW 85312 ) and $+1.65 \pm 0.50 \%$ o (PCA 91084). There is no systematic trend between sulfate $\delta^{34} S$ and abundance (Table 2 and 3), although PCA 91084 has both the highest $\delta^{34} S$ and the highest sulfate content $(1.75 \pm 0.50 \%$ and $12900 \pm 100$ ppm S, respectively). PCA 91084 is the only heated CM in our suite 294 (Alexander et al., 2012, 2013). If PCA 91084 is excluded, the CM sulfate $\delta^{34}$ S average becomes $-1.39 \pm 1.28 \%$ o $295(1 \sigma, n=12)$, which is the lowest across the $\mathrm{S}$ pools in CMs. The CM average $\Delta^{33} \mathrm{~S}$ for sulfate is $296+0.036 \pm 0.084 \%$ o $(1 \sigma, \mathrm{n}=13)$, with values ranging between $-0.036 \pm 0.008 \%$ (LEW 87022) and $297+0.263 \pm 0.008 \%$ o (Murchison). For $\Delta^{36}$ S, the CM sulfate average is $-0.16 \pm 0.15(1 \sigma, n=13)$, with values 298 ranging between $-0.33 \pm 0.25 \%$ (TIL 91722) and $+0.26 \pm 0.25 \%$ (MCY 05230). Whereas the sulfate 
component of PCA 91084 has an extreme $\delta^{34} S$ compared other CMs, its $\Delta^{33} \mathrm{~S}$ and $\Delta^{36} \mathrm{~S}$ values are $+0.016 \pm 0.008 \%$ and $-0.15 \pm 0.25 \%$ respectively, i.e., within the range of the other CMs.

301 The average $\delta^{34} S$ for $S^{0}$ is $+3.06 \pm 1.63 \%$ o $(1 \sigma, n=13)$ relative to $C D T$, with values ranging between 302 1.84 $\pm 0.15 \%$ o (PCA 91084) and $+5.19 \pm 0.15 \%$ (LEW 87022), representing a $\sim 7 \%$ range. When all CMs are 303 considered, no trend appears between the $S^{0} \delta^{34} S$ values and contents. If PCA 91084 (the only heated CM) is 304 excluded, the average becomes $+3.46 \pm 0.74 \%(1 \sigma, n=12)$, with the minimum value displayed by ALH 30584029 at $+2.32 \pm 0.15 \%$ o ( $\sim 3 \%$ o total range only). Elemental S is the S-bearing phase with the highest 306 average $\delta^{34} \mathrm{~S}$. The average $\Delta^{33} \mathrm{~S}$ is $+0.016 \pm 0.113 \%$, with values ranging between $-0.104 \pm 0.008 \%$ (LEW 307 87022) and $+0.256 \pm 0.008 \%$ (MCY 05230). While the $S^{0}$ pool is the least abundant S-bearing phase in our $308 \mathrm{CMs}$, it is the pool with the highest $\Delta^{33} \mathrm{~S}$ variability. The $\Delta^{36} \mathrm{~S}$ average is $+0.159 \pm 0.172 \% 0(1 \sigma, \mathrm{n}=13)$, with 309 values ranging from $-0.23 \pm 0.25 \%$ ( 310 sulfate, $\mathrm{S}^{0}$ in PCA 91084 has an extreme $\delta^{34} \mathrm{~S}$ compared other CMs, but its $\Delta^{33} \mathrm{~S}$ and $\Delta^{36} \mathrm{~S}$ values, of $311+0.045 \pm 0.008 \%$ and $-0.23 \pm 0.25 \%$, respectively, are, within uncertainties in the range of the other CMs.

312 In comparison with Murchison and MCY 05230, all our meteorites lie relatively close to the origin in a $313 \Delta^{33} \mathrm{~S}$ vs. $\Delta^{36} \mathrm{~S}$ plot. In detail, they display $\Delta^{33} \mathrm{~S}$ values ranging between indistinguishable from CDT to 314 significantly negative (Fig. 4 and 5). The data form a rough array with a slope of $-3.9 \pm 2.2$ in the $\Delta^{33} \mathrm{~S}$ vs. $\Delta^{36} \mathrm{~S}$

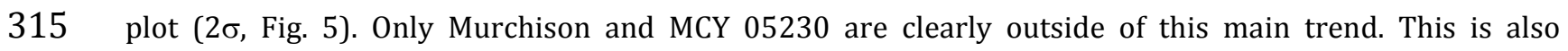
316 observed for their bulk S (Fig. 3b), and is a result of their sulfide, sulfate and S0 extracts all showing large 317 positive $\Delta^{33}$ S associated with near-zero $\Delta^{36}$ S (Fig. 5). Again, no systematic S isotope differences were 318 observed between falls and finds.

\subsection{Duplicates}

322 of our whole S extraction protocol (Table 4). Two and three replicates were produced for Murchison and 323 Cold Bokkeveld, respectively. Whereas the sulfate extractions yielded S contents that were consistent 324 within $10 \%$ between replicates (and indistinguishable isotope compositions), the $\mathrm{S}^{0}$ and sulfide extractions 325 showed variations of up to roughly a factor of two. The $\delta^{34} \mathrm{~S}$ values of the sulfide duplicates for Murchison 
326 remain close, $-0.02 \pm 0.30 \%$ ( $5700 \mathrm{ppm} \mathrm{S}$ ) and $+0.30 \pm 0.30 \%$ (10800 ppm S), despite the factor of two 327 difference in sulfide $S$ contents. In contrast, $\Delta^{33} \mathrm{~S}$ variations for sulfide $\mathrm{S}$ are outside of four times the 328 estimated analytical uncertainty, with values of $+0.136 \pm 0.010 \%$ o $(5700 \mathrm{ppm} \mathrm{S})$, and $+0.177 \pm 0.010 \%$ o

329 (10800 ppm S). The $\delta^{34} \mathrm{~S}$ values of $\mathrm{S}^{0}$ are resolvable across the two Murchison duplicates, being $330+4.62 \pm 0.15 \%$ o (1100 ppm S) and $+2.59 \pm 0.15 \%$ (2100 ppm S). In contrast, their $\Delta^{33 S}$ values are 331 indistinguishable from one another, being $+0.236 \pm 0.008 \%$ o (1100 ppm S) and $+0.247 \pm 0.008 \%$ o $(2100 \mathrm{ppm}$ 332 S). These variations are significant but remain lower than the overall variations between Murchison and 333 other CMs. In contrast with Murchison, no $\Delta^{33}$ S variability was resolved between Cold Bokkeveld duplicates 334 (Table 4).

336 5. Discussion.

\section{$337 \quad 5.1$ Evaluation of our S-extraction protocol}

$338 \quad$ 5.1.1 Sulfate

339 Gao and Thiemens (1993) report the sulfate contents of two pieces of Murchison (Field Museum, 340 Chicago), one piece of ALH 84029, and one piece of Mighei. They extracted $6900 \mathrm{ppm}$ S as sulfate from 341 Mighei, which is similar to the range of sulfate concentrations observed in our CM extractions. The 342 extractions by Gao and Thiemens (1993) of sulfate from two pieces of Murchison yielded 5600 and 6000 343 ppm S, which are indistinguishable from our extractions for this meteorite (Table 2). Their extraction of 344 sulfate from ALH 84029 yielded 8000 ppm S, which is nearly twice the amount that we extracted (4800 $345 \mathrm{ppm}$ ). Burgess et al. (1991) used combustion techniques to determine sulfate contents in Murchison, 346 Mighei, Nogoya and Murray. They report $14100 \mathrm{ppm} \mathrm{S}$ as sulfate for Murchison, and $14500 \mathrm{ppm}$ S as sulfate 347 for Nogoya, both of which are much higher than we obtained from our samples (Table 1). These 348 discrepancies could indicate sample heterogeneity in the sulfate distribution, as reported for carbonate 349 abundances in these samples (e.g., Alexander et al., 2015). Alternatively, they could reflect the extraction of 350 tochilinite (in which S is present as a sulfide) by Burgess et al. (1991) together with sulfate, as suggested by 351 these authors. Tochilinite mineral could account for more than $50 \%$ of the S released at the temperature 
assigned for sulfate (Burgess et al., 1991), possibly explaining the roughly factor of two of difference between our data and the combustion data.

\subsubsection{Elemental S}

Burgess et al. (1991) report $S^{0}$ contents of 1500 ppm in Murchison, and 900 ppm for Nogoya using their stepped combustion approach. These values compare well with our extractions, being $1600 \pm 100 \mathrm{ppm}$ and $1400 \pm 100$ ppm S, respectively (Table 1). Note that Burgess et al. (1991) also reported 600 ppm S0 for both

359 Mighei and Murray, in the range of our CM extractions that averaged 1900 $\pm 1400 \mathrm{ppm}$ S. Gao and Thiemens 360 (1993) used $\mathrm{CCl}_{4}$ as a solvent to extract $\mathrm{S}^{0}$, and they report $\mathrm{S}^{0}$ contents that were typically $<500 \mathrm{ppm} \mathrm{S}$. We do not think that the higher $S^{0}$ contents we obtained compared to Gao and Thiemens (1993) results from an oxidation reaction during the extraction process (for instance from sulfide, or from an organic 363 polysulfur compound). Our extractions were performed under anoxic conditions in an $\mathrm{N}_{2}$ atmosphere. We 364 also note that water-soluble S-bearing organics would have been removed in the water traps before the $\mathrm{S}^{0}$ extraction (section 3.1). The discrepancy between our dataset and those of Gao and Thiemens (1993) therefore appears to be best explained by sample heterogeneity.

\subsubsection{Sulfide}

The extraction of sulfide was conducted with multiple steps. We first extracted the acid-volatile S fraction (AVS) by digesting the powdered samples in sub-boiling $6 \mathrm{~N} \mathrm{HCl}$. After the extractions, all the solutions displayed a yellow coloration, suggesting the presence of dissolved $\mathrm{Fe}^{3+}$. Iron speciation is poorly characterized in $\mathrm{CM}$, but $\mathrm{Fe}^{3+}$ is thought to be ubiquitous, hosted in magnetite and cronstedtite (e.g., Zolensky et al., 1997; Beck et al., 2012; Sutton et al., 2013; Howard et al., 2015). Reaction between reduced S and ferric iron can occur in acidic solution (Pruden and Bloomfield, 1968; Rice et al., 1993), producing S $^{0}$

375 as follows:

$$
2 \mathrm{Fe}^{3+}+\mathrm{H}_{2} \mathrm{~S} \rightarrow 2 \mathrm{Fe}^{2+}+\mathrm{S}^{0}+2 \mathrm{H}^{+}
$$

$\mathrm{S}^{0}$ produced by reaction 1 can be recovered by the use of $\mathrm{CrCl}_{2}$ solution (Canfield, 1986; Mayer and Krouse, 2004). We extracted the $S^{0}$ in solution (step 12) and the $S^{0}$ fraction that had precipitated (step 13).

379 Because $S^{0}$ is poorly soluble in acidic environments, $S$ in step 13 is more abundant than in step 12. AVS 
represents only $\sim 7 \%$ of the extracted sulfide pool, whereas steps 12 and 13 represent $\sim 4 \%$ and $\sim 89 \%$, respectively. If correct, most of the sulfide is oxidized to $S^{0}$ during the AVS extraction, presumably due to the large $\mathrm{Fe}^{3+}$ contents in the studied samples. AVS $\Delta^{33} \mathrm{~S}$ values are compared to those of step 12 and step

38313 in Fig. S2, and compared with the naturally occurring S0 (i.e., step 2) in Fig. S3. The AVS, step 12 and step

38413 isotopic compositions have, to first order, indistinguishable $\Delta^{33} \mathrm{~S}$ for most CMs (see detailed discussion 385 in Appendix A). When compared with step 2, the data show considerable scatter, and do not fall on a 1:1 slope. Despite the lack of a clear $\delta^{34} \mathrm{~S}$ relationship, this further supports the conclusion that AVS, step 12 and step 13 are linked through sulfide oxidation during the AVS extraction (reaction 1 ). The differences between the step 2 and sulfide pools (Fig. S3) indicate that our protocol results in minimal contamination of the sulfide-derived $S$ by the naturally occurring $S^{0}$.

\subsubsection{Bulk S and sulfide contents of CMs: Comparison with previous work}

Our observations suggest that most of the sulfide pool is oxidized during the AVS step and recovered in step 13. If not recovered properly, this might lead to an underestimation of both the sulfide content and the bulk S content of chondrites. We suggest that this accounts for the discrepancy between our study and Gao and Thiemens (1993) who reported bulk S contents for three CMs $\leq 1$ wt.\%, $~ 75 \%$ of which is in sulfate. This is in contrast to the bulk S contents in our 13 CMs that range from 1.7 wt.\% to 3.0 wt.\%, with sulfide making up at least half the bulk S in most of them (Table 1). Gao and Thiemens (1993) recovered sulfide by digesting their chondrites in $\mathrm{HCl}$, and only considered their AVS extractions as sulfide-derived S. Using this ppm in our study.

401 Burgess et al. (1991) and Dreibus et al. (1995) also determined S abundances in several CM falls. 402 Burgess et al. (1991) reported an average S content of 2.99 \pm 0.31 wt.\% S $(1 \sigma)$ for four CMs (Murchison, 403 Mighei, Nogoya, and Murray) and ranging from 2.65 wt.\% to 3.38 wt.\% S. Dreibus et al. (1995) report a 404 comparable average bulk content of 3.26 $\pm 0.68 \mathrm{wt} \%(1 \sigma)$ for eleven CMs, including three falls (Murchison, 405 Cold Bokkeveld, and Nogoya) with individual bulk S contents varying between 2.20 wt.\% and 4.90 wt.\% S. 406 Our bulk S contents for the same samples are lower by 20-35\%. Burgess et al. (1991) and Dreibus et al. 
(1995) both used combustion techniques and, therefore, likely accessed organic S. This could at least partially explain the differences in reported bulk S contents as only inorganic $\mathrm{S}$ was extracted with our protocol. Sulfur in soluble organic matter is likely to be $<1 \%$ of the $S$ budget (Cooper et al., 1997). The $S$ content of insoluble organic matter is $\sim 10 \pm 5 \mathrm{wt} . \%$ (Alexander et al., 2007), representing $\sim 0.1 \mathrm{wt.} \% \mathrm{~S}$ in

411 the bulk rock or $\sim 5 \%$ of the $\mathrm{S}$ budget in CMs. Thus, organically bonded $\mathrm{S}$ is only a partial explanation for the differences in bulk $\mathrm{S}$ contents reported here and the two earlier studies. If there were any sulfates that were insoluble in water (e.g., $\mathrm{BaSO}_{4}$ and/or jarosite), these would not have been extracted by our protocol

414 (section 3.1). If so, the $20-30 \%$ lower $\mathrm{S}$ content associated with our technique relative to combustion 415 studies (Burgess et al. 1991) would suggest that about half of the CMs sulfates would not be extracted by our technique. We cannot exclude this possibility, and systematic studies comparing bulk S data obtained by wet chemistry to combustion extractions on the same batches of powder are needed. Finally, sample

418 heterogeneity is also a possible explanation, particularly when samples come from different stones of the 419 same fall. Our replicate extractions on aliquots of the same Cold Bokkeveld and Murchison powdered samples yielded bulk S contents that varied by roughly a factor of 2 (Table 4). Bulk S contents reported by a number of other authors also show factors of two variations for a given chondrite (Mason 1963; Kaplan and 422 Hulston, 1966; Fitzgerald and Jacques, 1982; Kinnunen and Saikkoken, 1983; Graham and Yanai, 1985; 423 Jarosewitch, 1971). The only puzzle with this explanation is why, for the same meteorites, all our samples should have lower S contents than reported by Burgess et al. (1991) and Dreibus et al (1995).

\subsubsection{Sulfur isotope heterogeneity in different pieces of the Murchison meteorite}

427 The comparison of our isotopic measurements of S from the British Natural History Museum sample of Murchison (BM.1988,M23) and the measurements by Gao and Thiemens (1993) (ME 2641) reveals a striking difference in the $\Delta^{33} S$ and provides evidence for significant $S$ isotope heterogeneity in the

430 Murchison material. This heterogeneity may reflect the fact that Murchison is a breccia containing 431 xenolithic fragments of several other meteorite types, which are millimeter-to-centimeter-sized (e.g., Fuchs 432 et al., 1973; Benedix et al., 2015).

433 For the Murchison splits reported by Gao and Thiemens (1993), all the S components have near zero $434 \Delta^{33} \mathrm{~S}$ values. In contrast, we observe strongly positive $\Delta^{33} \mathrm{~S}$ values in the three components extracted from 
435 our Murchison splits. It requires the redistribution of S-bearing phases along parent body processes (if any redistribution at all) to be limited to a scale below the meter scale (i.e. below what is sampled between

437 Murchison rocks in different museums).

\subsection{Exploring the genetic link between $S^{0}$ and sulfate}

Based on their S isotopic composition, we develop the idea that sulfates in CMs result from parent body processing. The thermodynamic modeling of Zolensky et al. (1989) suggested that the Eh-pH conditions

442 prevailing during aqueous alteration on the CM parent body would not allow for oxidation of sulfide to 443 sulfate. However, Airieau et al. (2005) reported the occurrence of non-terrestrial $\Delta^{17} \mathrm{O}$ for sulfate in $6 \mathrm{CMs}$

444 falls (including Murchison, Cold Bokkeveld, Nogoya and Banten). This observation demonstrates that the sulfate formation occurred on the parent body, with extraterrestrial water, as opposed to sulfates forming on Earth.

447 If the only heated CM, PCA 91084, is excluded, sulfate has an average $\delta^{34} \mathrm{~S}$ of $-1.38 \pm 1.28 \% 0(1 \sigma, \mathrm{n}=12)$.

448 This is systematically lower than the $\mathrm{S}^{0}$ average of $3.56 \pm 0.74 \%$ o $(1 \sigma, \mathrm{n}=12$ PCA 91084 excluded $)$ or than the 449 sulfide average of $-0.17 \pm 0.33 \%(1 \sigma, n=12)$. Despite some overlap, the $\delta^{34} S$ of sulfate is generally lower 450 than for sulfide, and is systematically lower than for $S^{0}$ in a given CM. Similar observations were made in 451 the pioneering study of a single chip of Orgueil (CI) by Monster (1965), as well as on Cold Bokkeveld, 452 Mighei and Murray (Kaplan and Hulston, 1966). Gao and Thiemens (1993) also report low $\delta^{34}$ S sulfate 453 values relative to $\mathrm{S}^{0}$ or sulfide in their CMs. If sulfates were formed by oxidation of a more reduced 454 precursor (sulfide or $\mathrm{S}^{0}$ ), these $\mathrm{S}$ isotope data would indicate an oxidation process that preferentially 455 incorporates ${ }^{32} \mathrm{~S}$ into the sulfate, producing isotopically light sulfate. This is consistent with a normal kinetic isotope effect associated with oxidation like those that have been observed in the oxidation of 457 aqueous sulfide (principally HS; e.g., Fry et al., 1988) and sulfide minerals (FeS; e.g., Lewis and Krouse, 458 1968).

459 Depending on the size of the studied reservoirs, resolvable ${ }^{34} \mathrm{~S}$ enrichments are expected in the residual precursor. Sulfate represents on average $25 \pm 12 \%$ of the bulk $S$ whereas sulfide and $\mathrm{S}^{0}$ represent $65 \pm 15 \%$ and $9 \pm 7 \%$ respectively (section 4.2). Assuming closed system (Bland et al., 2009) and that the sulfate 
462 precursor (sulfide or $\mathrm{S}^{0}$ ) had a $\delta^{34} \mathrm{~S}$ equal to $\mathrm{CDT}$, the roughly $1 \%{ }^{34} \mathrm{~S}$-depletion in sulfate relative to CDT

463 should be compensated by a roughly 0.4 or $2.5 \%{ }^{34}$ S-enrichment in the sulfide or $\mathrm{S}^{0}$ pool, respectively.

464 Sulfide and $S^{0}$ have average $\delta^{34} S$ values of $-0.15 \pm 0.33 \%$ and $+3.46 \pm 0.74 \%$ o $(1 \sigma)$, respectively. Within

465 uncertainty, both mechanisms could be reasonable explanations, and on the basis of this ${ }^{34} \mathrm{~S}$ mass balance,

466 sulfate could be formed by both oxidation of sulfide or $\mathrm{S}^{0}$.

467 Sulfates in chondrites could have been produced on Earth, by reaction of sulfides (and/or elemental S)

468 with atmospheric oxygen. For example, Gounelle and Zolensky (2001) report clear evidence for sulfate

469 veins formation in Orgueil (CI chondrite) during the storage history of the samples. This is consistent with

470 the Orgueil sulfate $\Delta^{17} \mathrm{O}$ value of $-0.12 \pm 0.20 \%$ ( $2 \sigma$, Airieau et al., 2005), i.e. indistinguishable from sulfates

471 formed on Earth (with a $\Delta^{17} \mathrm{O}$ value of $0.00 \%$, Airieau et al. 2005). This could indicate that mostly

472 terrestrial oxygen was involved in the formation of these sulfates (Airieau et al., 2005). On the other hand,

473 CMs have sulfate with variable $\Delta^{17} \mathrm{O}$ values, between $+1.18 \pm 0.20 \%$ and $-0.23 \pm 0.20 \%$. These values are

474 clearly not terrestrial, indicating that sulfates in CMs, in contrast to CIs, were mostly produced in their

475 parent body during late stage aqueous alteration (Airieau et al., 2005). Interestingly, the bulk $\Delta^{17} \mathrm{O}$ values

476 for CMs is between $-0.67 \%$ and $-3.07 \%$ (Clayton and Mayeda, 1999), always significantly lower than the

477 corresponding sulfate values (Airieau et al., 2005). In the canonical water/rock interaction model (Clayton

478 and Mayeda, 1999), this would indicate that the sulfates were produced early in the aqueous alteration

479 sequence, before substantial isotopic exchange between water (presumably carrying a high $\Delta^{170}$ value) and

480 the anhydrous silicates (presumably carrying a low $\Delta^{17} \mathrm{O}$ value) had occurred (Clayton and Mayeda, 1999).

481 Independent of when and where the chondritic sulfates were produced, the question of their precursor

482 (sulfide, $\mathrm{S}^{0}$, or another S-bearing phase) remains elusive, at best, in the literature. Petrographic analyses of

483 CMs report alteration of sulfides (e.g., Zolensky et al., 1997; Rubin et al., 2007), suggesting that sulfide

484 oxidation will contribute to the chondritic sulfate budget. However, sulfates always display $\Delta^{33} \mathrm{~S}$ anomalies

485 that are significantly larger than sulfide (Fig. 7). Since sulfide oxidation would only transfer the sulfide $\Delta^{33 S}$

486 to the sulfates, the mismatch between sulfates and sulfides $\Delta^{33} \mathrm{~S}$ values indicate that sulfides cannot be the

487 main precursor of sulfate. On the other hand, sulfate and $S^{0}$ have similar $\Delta^{33} S$ values (Fig. 7). The slope

488 observed between $\Delta^{33} S$ values of $S^{0}$ and sulfate is $0.86 \pm 0.24(2 \sigma)$ with an intercept of $0.028 \pm 0.018 \%$. At 
first order, this slope is statistically indistinguishable of a 1:1 slope, allowing sulfate to be produced by $\mathrm{S}^{0}$ oxidation, not sulfide oxidation. This is not true for the sulfates from MCY 02530 that have a $\Delta^{33}$ S value that is indistinguishable from the sulfides of this $\mathrm{CM}$, and significantly different from that of $\mathrm{S}^{0}$. This $\mathrm{CM}$ is 492 excluded from the following discussion.

493 Although sulfates and $S^{0}$ have similar $\Delta^{33} S$ values, sulfates always seem to be biased toward slightly 494 higher $\Delta^{33} S$ values than $S^{0}$ at the $\sim 0.020-0.060 \%$ level (averaging at $+0.036 \pm 0.042 \%$, $2 \sigma$, versus a total 495 range of $\sim 0.300 \%$ ). This is evident in Table 3, Fig. 7A and 9. This slightly higher $\Delta^{33} \mathrm{~S}$ value in sulfate, relative to $S^{0}$, is correlated to the ${ }^{34} S$ difference between sulfate and $S^{0}$ : The more sulfates are depleted in ${ }^{34} \mathrm{~S}$ relative to $\mathrm{S}^{0}$, the higher their $\Delta^{33} \mathrm{~S}$ are relative to $\mathrm{S}^{0}$ (Fig. 9). In addition, sulfates show a slight $\Delta^{36} \mathrm{~S}$ depletion relative to $S^{0}$, averaging at $-0.31 \pm 0.26 \%(2 \sigma)$. However, the relative $\Delta^{36} S$ uncertainties are significantly larger than for $\Delta^{33} \mathrm{~S}$ (table 3), preventing any direct trend with ${ }^{34} \mathrm{~S}$ values to be observed

500 (Table 3). This is nevertheless the first time that the relationship observed in Fig. 9 is observed in natural 501 samples, and we suggest that it reflects the specific nature of the $\mathrm{S}^{0}$ oxidation mechanism to sulfate. The mass dependence of an isotope fractionation accompanying a chemical reaction or physical process among more than two stable isotopes is typically given by a power law relationship, e.g., ${ }^{33} \alpha=\left({ }^{34} \alpha\right)^{\wedge} \theta$ where $\alpha$ is the fractionation factor (Craig, 1957; Hulston and Thode, 1965; Matsuhisa et al., 1978; Clayton and Mayeda, 1996; Young et al., 2002). By convention, the reference exponent $\theta$ for the relationship between ${ }^{33} \alpha$ and ${ }^{34} \alpha$

506 is taken to be 0.515 , the approximate exponent of mass dependence describing most simple equilibrium 507 isotope exchange reactions (Matsuhisa et al., 1978; Young et al., 2002). Rare exceptions to this rule have 508 been observed, particularly for complex kinetic processes, where exponents of mass dependence differing 509 significantly from the reference exponent of 0.515 were observed and coined "non-canonical" effects (Eiler 510 et al., 2013). For those fractionations, $\Delta^{33} \mathrm{~S}$ is predicted to depart from a reference across a given process 511 and to be correlated with $\delta^{34} \mathrm{~S}$ variations. In our case, a mechanism linking $\mathrm{S}^{0}$ and sulfate would require that 512 the ${ }^{33} \mathrm{~S} /{ }^{32} \mathrm{~S}$ fractionation be $0.505 \pm 0.005$ times that of the ${ }^{34} \mathrm{~S} /{ }^{32} \mathrm{~S}$ fractionation.

\section{$515 \quad$ 5.3. Sulfur cosmochemistry}


516 The analyses presented here yield a much larger range of S-MIF in the major S-carrying phases than 517 previously recognized in chondrites. Gao and Thiemens (1993) may have missed identifying the signature 518 because the number of CM chondrites that they studied was small $(n=3)$. Repeat analyses of the same 519 meteorites in some cases match well within uncertainty with Gao and Thiemens (1993), but we report a 520 distinctly different bulk S isotope composition for Murchison, suggesting sample heterogeneity. The largest 521 S-MIF signals are found in $\mathrm{S}^{0}$, but they are also preserved in sulfide and sulfate - the latter being a water522 soluble phase.

523 Mixing of S from diverse nucleosynthetic origins, such as that associated with ${ }^{32}$ S-bearing SiC attributed 524 to decay of ${ }^{32} \mathrm{Si}$ produced in supernovae, is not a good mechanism for explaining the observed S isotope 525 anomalies in CMs. SiC grains comprise 30 $\pm 15 \mathrm{ppm}$ of CMs (Davidson et al. 2014), and of these, the grains 526 carrying ${ }^{32} \mathrm{~S}$-excesses $\left(\Delta^{33} \mathrm{~S}\right.$ values down to $\left.-500 \%\right)$ are rare $(\sim 1-5 \%$ - Hoppe et al., 2012; Fujiya et al., 527 2013; Pignatari et al., 2013). Considering the S abundance of CMs, ${ }^{32}$ S addition by these grains would only 528 raise the ${ }^{32} \mathrm{~S} /{ }^{34} \mathrm{~S}$ ratio by $\sim 5 \times 10^{-5} \%, \sim 3$ to 5 orders of magnitude too low to account for the $\Delta^{33} \mathrm{~S}$ variations 529 in $\mathrm{S}^{0}$ and sulfide, respectively.

530 The significant non-mass dependent $(>0.1 \% 0){ }^{33} \mathrm{~S}$ variations in phases that condense at different 531 temperatures suggest a photochemical process. During its T-Tauri phase, the young Sun would have 532 emitted significantly greater deep and extreme UV than it does today (Zahnle and Walker, 1982). This UV 533 radiation would have been available for photolysis in the transparent hotter inner parts of the solar nebula 534 (Rai et al. 2005) and along unshaded parts of the surface of the disk, as modeled by Ciesla (2010) and Ciesla 535 and Sandford (2012). Any produced photochemical S-isotopic signature could have been transferred to 536 solids via condensation of S-bearing phases, followed by transportation to the midplane of the disk by 537 vertical mixing (Ciesla, 2010) where it would have been accreted as part of the chondrite matrices.

538 For the present CM S isotope data, the carrier of the anomaly appears to be related to $\mathrm{S}^{0}$ or an $\mathrm{S}^{0}$ 539 precursor rather than a high temperature sulfide condensate, as was suggested in prior studies (Rai et al., 540 2005; Rai and Thiemens, 2007; Antonelli et al., 2014), but the production mechanisms of the $\mathrm{S}^{0}$ or $\mathrm{S}^{0}$ 541 precursor remains unclear. The S-MIF signature could reflect direct photochemical production of $\mathrm{S}^{0}$, 542 provided that sufficient UV were available in the cooler parts of the disk where $\mathrm{S}^{0}$ could condense or be 543 captured by grain surfaces. 
544 The observation that $\Delta^{33} \mathrm{~S}$ values of $\mathrm{S}^{0}$ (which is water-insoluble) is correlated with values in water-

545 soluble phases (such as sulfate), in combination with the observation that different pieces of the same 546 meteorite preserve evidence for variable $\Delta^{33} \mathrm{~S}$ values (Murchison) implies that the S-MIF signal is variable

547 on a sub-meter scale at least in Murchison. The corollary is that parent body aqueous processing and flow

548 was not sufficient to homogenize the isotopic signals at this scale. This argues against a pervasive fluid

549 flow in the regions where the CM chondrites were formed.

550 PCA 91084, the only heated CM analyzed here (Alexander et al., 2007, 2013), displays国国 chemistry

551 pattern that differs from the other CMs. We suggest that this may reflect oxidation during heating in the CM

552 parent body (Appendix B). Further study of heated carbonaceous chondrites is needed to validate this 553 hypothesis.

554

\section{4 $\mathrm{H}_{2} \mathrm{~S}$ Photochemistry as a candidate production mechanism for S-MIF}

$556 \mathrm{H}_{2} \mathrm{~S}$ photochemistry is considered as a candidate for the S-MIF production because it is thought to be 557 the dominant S-bearing gas phase in the solar nebula (Chakraborty et al., 2013). $\mathrm{H}_{2} \mathrm{~S}$ absorbs in several regions in the UV, with a broad absorption feature extending from $\sim 317 \mathrm{~nm}$ to $\sim 165 \mathrm{~nm}$, and a series of banded absorption features that extend from $\sim 165 \mathrm{~nm}$ down to $\sim 40 \mathrm{~nm}$ with a peak at $\sim 90 \mathrm{~nm}$. This peak

560 includes several bands around Lyman alpha wavelengths at $121.6 \mathrm{~nm}$ (i.e., the most intense T-Tauri 561 wavelengths, Zahnle and Walker, 1982, Okabe et al. 1978). The longer wavelength feature appears to be 562 associated with the production of atomic hydrogen and $\mathrm{SH}$, while the shorter wavelength region is associated with the production of atomic $\mathrm{H}$ and S (Okabe, 1978).

564 Farquhar et al. (2000b) report isotopic fractionations associated with photolysis experiments that 565 access the longer wavelength $(\sim 317 \mathrm{~nm}$ to $\sim 165 \mathrm{~nm})$ absorption feature using continuum radiation extending from $\sim 200 \mathrm{~nm}$ to the visible. Recently, Chakraborty conducted a series of photolysis experiments of $\mathrm{H}_{2} \mathrm{~S}$ by UV radiation with wavelengths of $90,121.6,139.1$, and $157 \mathrm{~nm}$ to investigate isotope

568 effects in the shorter wavelength region. These experiments indicate that photolysis at different 569 wavelengths produces $\mathrm{S}^{0}$ and residual $\mathrm{H}_{2} \mathrm{~S}$ with different types of isotopic signals, most notably $\Delta^{36} \mathrm{~S} / \Delta^{33} \mathrm{~S}$ 570 ratios that vary as a function of the wavelength of the incident UV. The experiments undertaken by 571 Farquhar et al. (2000b) yielded positive $\Delta^{33} \mathrm{~S}$ values in the produced $\mathrm{S}^{0}$, where products and residues lie on 
572 a positive $\Delta^{36} \mathrm{~S} / \Delta^{33} \mathrm{~S}$ array $(\sim+1.7 \pm 1.5,2 \sigma$, see Farquhar et al., $2000 \mathrm{~b})$. On the other hand, the photolysis 573 experiments of Chackraborty et al. (2013) undertaken at discreet wavelengths of $122 \mathrm{~nm}$ and $139 \mathrm{~nm}$ 574 produced negative $\Delta^{33} S$ values in $S^{0}$ down to $-1.54 \pm 0.02 \%$, and a $\Delta^{36} S / \Delta^{33} S$ ratio of $-2.77 \pm 0.46(2 \sigma)$. 575 Experiments conducted at $90 \mathrm{~nm}$ and $157 \mathrm{~nm}$ yielded $S^{0}$ with positive $\Delta^{33} \mathrm{~S}$ values and near-zero $\Delta^{36} \mathrm{~S}$ 576 values, yielding $\Delta^{36} \mathrm{~S} / \Delta^{33} \mathrm{~S}$ values of $+1.4 \pm 0.2$ and $+0.1 \pm 0.2$, respectively (Chakraborty et al., 2013). Note 577 that there are no systematic $\delta^{34} \mathrm{~S}$ variations between the different mass independent signatures in the 578 experiments (Chakraborty et al., 2013). Thus, as opposed to non-canonical mass dependant fractionations 579 (section 5.2, Eiler et al. 2013), no systematic $\delta^{34}$ S variations can be a priori expected to correlate with the 580 MIF signatures.

581 In CMs, most $S^{0}$ extracts have homogeneous $\delta^{34} S$, negative $\Delta^{33} S$ values and lie on a $\Delta^{36} S / \Delta^{33} S$ slope of $5823.1 \pm 1.0(-3.9 \pm 2.2$ when sulfide and sulfate extracts are taken into account; $2 \sigma$; Fig. 4a and 5). This 583 relationship is similar to that seen in the experimental results in the Lyman alpha region (Chakraborty et 584 al., 2013). Given that this is one of the most intense emission regions in the far ultraviolet of T-tauri stars 585 (Zahnle and Walker, 1982) we suggest a link to the observation of S-MIF in the CM chondrites.

The positive $\Delta^{33} \mathrm{~S}$ values for $\mathrm{S}^{0}$ of Murchison and MCY 05230 are associated with near-zero $\Delta^{36} \mathrm{~S}$ values, or $\Delta^{36} \mathrm{~S} / \Delta^{33} \mathrm{~S}$ values of $+0.9 \pm 0.1$ and $+1.3 \pm 0.1$, respectively, suggest a different mechanism. Magnetic isotope effects, producing large $\Delta^{33} \mathrm{~S}$ anomalies associated with negligible $\Delta^{36} \mathrm{~S}$ variations, could explain these values. It was experimentally shown that S magnetic isotope effects can be produced in highly specific 590 chemical conditions during S reduction processes (Oduro et al. 2011). This process leads to the 591 preferential partitioning of ${ }^{33} \mathrm{~S}$ into the products of the reaction (sulfides), leaving behind ${ }^{33} \mathrm{~S}$-depleted sulfates. In the CMs the sulfates, sulfides and $\mathrm{S}^{0}$ all share positive $\Delta^{33} \mathrm{~S}$ anomalies, leaving unexplained what 593 the complement of these isotopic reservoirs would be. It is also unclear why only two of the CMs would 594 have experienced processes allowing the expression of magnetic isotope effect, without affecting any other 595 meteorites. We here argue that the positive $\Delta^{36} \mathrm{~S} / \Delta^{33} \mathrm{~S}$ ratios can also result from a photodissociation 596 process, since several $\mathrm{H}_{2} \mathrm{~S}$ photodissociation experiments show positive $\Delta^{36} \mathrm{~S} / \Delta^{33} \mathrm{~S}$ ratios, including those 597 conducted at both shorter and longer wavelengths than the Lyman alpha wavelengths (Chakraborty et al., 598 2013). Considering that absorption by gas phase species such as $\mathrm{H}_{2}, \mathrm{CO}, \mathrm{CH}_{4}, \mathrm{~N}_{2}, \mathrm{NH}_{3}$ would preferentially 
shield shorter wavelengths (Keller-Rudeck et al., 2013), we suggest this change reflects a shift to longer wavelengths after attenuation of Lyman alpha by absorption in the nebula (See Fig. 8). Importantly,

601 variations of $\Delta^{36} \mathrm{~S} / \Delta^{33} \mathrm{~S}$ from sample to sample (Fig. 5) resulting from the photodissociation processes 602 provide evidence for heterogeneous accretion of S-bearing phases by the parent body of CMs. This rules out 603 parent body processes as the cause for these $\mathrm{S}$ isotope variations (through for example the heterogeneous 604 redistribution of some S-MIF-bearing phase after accretion).

\section{5}

606

\subsubsection{Model A: sulfides and $S^{0}$ are both direct condensates.}

608 One possibility for explaining the S isotope variations in CMs is that when temperatures in the nebula 609 were low enough, any photolytic $\mathrm{S}^{0}$ carrying S-MIF condensed and the remaining $\mathrm{H}_{2} \mathrm{~S}$ sulfidized metal to 610 form sulfides following reaction 2 (Zolensky et al., 1995; Lauretta et al., 1996, 1998).

$$
\mathrm{Fe}^{0}+\mathrm{H}_{2} \mathrm{~S}=\mathrm{FeS}+\mathrm{H}_{2}(2)
$$

612 Metal sulfidation experiments show that the reaction 2 leads to the formation of troilite (FeS) at $\sim 700$

$613 \mathrm{~K}$ (Lauretta et al., 1996). At a given $\mathrm{H}_{2} \mathrm{~S}$ content, the sulfidation rate decreases dramatically with 614 temperature, but this mechanism remains efficient until temperatures are as low as $\sim 500 \mathrm{~K}$. Negative $\Delta^{33} \mathrm{~S}$

615 values in $\mathrm{S}^{0}$ are predicted to be associated with positive $\Delta^{33} \mathrm{~S}$ values in $\mathrm{H}_{2} \mathrm{~S}$ (recorded in sulfides), by mass 616 balance, as observed in $\mathrm{H}_{2} \mathrm{~S}$ photodissociation experiments (Chakraborty et al., 2013). Negative trends 617 between $\Delta^{33} \mathrm{~S}$ values for $\mathrm{S}^{0}$ and sulfides are hence predicted. However, a clearly positive trend is observed,

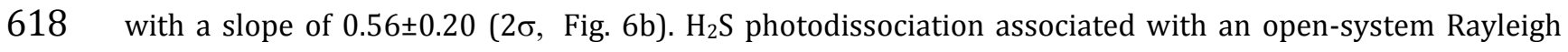
619 distillation process can reconcile the data with the experiments: Any $S^{0}$ would carry a negative $\Delta^{33} S$ value 620 relative to the residual $\mathrm{H}_{2} \mathrm{~S}$, without a significant change in $\delta^{34} \mathrm{~S}$ (Chakraborty et al. 2013). In an open621 system model, the photolytic $S^{0}$ would be distilled out of the system by condensation, continuously or in 622 discreet episodes, and deposited without substantial accumulation in the chondritic matrices. This would 623 enrich both the produced $\mathrm{S}^{0}$ and the remaining $\mathrm{H}_{2} \mathrm{~S}$ in ${ }^{33} \mathrm{~S}$. Assuming a bulk solar composition of $\Delta^{33} \mathrm{~S}=0$,

624 Rayleigh distillation is required to account for the occurrence of both positive and negative $\Delta^{33} \mathrm{~S}$ values for $625 S^{0}$ and sulfides (Fig. 8), as well as the observed positive correlation between sulfide and $S^{0}$ (Fig. 6b). 
626 Photolytic processes producing S-MIF under a Rayleigh distillation have been suggested elsewhere, as to occur in the Earth's upper atmosphere for $\mathrm{SO}_{2}$ photodissociation, explaining the $\mathrm{S}$ isotopic composition of 628 sulfate deposits in volcanic ashes from Antarctic ices (Baroni et al., 2007).

629 Quantitative modelling of $\mathrm{S}^{0}$ abundances and isotopic compositions in CMs remains challenging: 630 Experiments for $\mathrm{H}_{2} \mathrm{~S}$ photodissociation at $121.6 \mathrm{~nm}$ and $139.1 \mathrm{~nm}$ yielded $\Delta^{33} \mathrm{~S}$ fractionations between 631 produced $\mathrm{S}^{0}$ and residual $\mathrm{H}_{2} \mathrm{~S}$ ranging between $-1.54 \pm 0.02 \%$ and $-0.09 \pm 0.02 \%$ without systematic 632 changes in $\delta^{34} \mathrm{~S}$ (Chakraborty et al. 2013). A Rayleigh process involving these isotopic fractionations could 633 have produced the fractionations seen in all of the CM chondrites except Murchison and MCY 05230. For 634 the sake of illustration, we model an endmember case with constant $\Delta^{33} \mathrm{~S}$ fractionations and negligible $\delta^{34} \mathrm{~S}$ 635 fractionations (Fig. 8). The $\Delta^{33} \mathrm{~S}$ values for $S^{0}$ in CMs range between $-0.107 \pm 0.008$ and $+0.045 \pm 0.008 \%$, 636 which are consistent with a model where chondrites sampled $S^{0}$ produced by a Rayleigh process with a $6370.1 \% \Delta^{33} \mathrm{~S} \mathrm{~S}^{0}-\mathrm{H}_{2} \mathrm{~S}$ fractionation during $\mathrm{H}_{2} \mathrm{~S}$ photodissociation. If reaction 2 is ignored, the calculation yields 638 a fraction of remaining $\mathrm{H}_{2} \mathrm{~S}(f)$ between 0.99 and 0.30 to account for the range of $\mathrm{S}^{0}$ isotopic compositions in 639 all the CMs except Murchison and MCY 05230 (Fig. 8). Note that the ad hoc -0.1\%o value for the 640 fractionation is consistent with the $\sim 0.1 \pm 0.1 \%$ o difference generally observed between $S^{0}$ and sulfide in the 641 CMs (Table 3). A larger magnitude fractionation would reduce the required $f$ range needed to reproduce 642 the data. Using a fractionation of $-1.5 \%$ would require an $f<0.4$ to satisfy the observations, and all $\mathrm{S}^{0}$ 643 produced for $\mathrm{f}>0.4$ would have been lost.

644 CMs would be required to sample $S^{0}$ that underwent likely $<10 \%$ incremental accumulation after its 645 production, consistent with $S^{0}$ being the smallest $S$ reservoir in the CMs (Table 2). The amounts of $S^{0}$ 646 produced could be calculated after determining $f$ values for each chondrite. For example, GRA 98074 647 displays a $\Delta^{33} \mathrm{~S}$ value of $-0.055 \pm 0.008 \%$ or $\mathrm{S}^{0}$, which would correspond to an instantaneous product at a 648 fraction of residual $\mathrm{H}_{2} \mathrm{~S}(f)$ of 0.65 (Fig. 8). This isotopic value could also be produced by accumulation of all $649 \mathrm{~S}^{0}$ for $f$ values ranging between 0.7 and 0.6 (Fig. 8) or between 0.66 and 0.64 . The trade-off between these 650 possibilities is dictated by the acceptable amount of $S^{0}$ accreted in this chondrite to match the measured $\mathrm{S}^{0}$ 651 content $\left(1000 \pm 100\right.$ ppm S for GRA 98074, Table 2). For ALH 84029, its $S^{0}$ content is $5500 \pm 100$ ppm with a $652 \Delta \Delta^{33} S$ value of $+0.018 \pm 0.008 \%$ o (Table 2 and 3 ). This $\Delta^{33} S$ value would be reached for $f \approx 0.3$, but the higher 
$653 S^{0}$ content would require more $S^{0}$ accumulation. However, because the relative rate of photodissociation

$654\left(\mathrm{H}_{2} \mathrm{~S}->\mathrm{S}^{0}+\mathrm{H}_{2}\right.$, Fig. 8) to sulfidation $\left(\mathrm{H}_{2} \mathrm{~S}+\mathrm{Fe}^{0}=\mathrm{FeS}+\mathrm{H}_{2}\right.$, reaction 2$)$ is unknown, $f$ values cannot be

655 translated into $S^{0}$ concentrations. Nonetheless, this simple model qualitatively accounts for the absence of 656 direct trend between the $S^{0}$ isotopic composition and the amount of $S^{0}$ observed in a given CM (Table 2 and $6573)$.

658 The distinct $\Delta^{36} \mathrm{~S} / \Delta^{33} \mathrm{~S}$ values for Murchison and MCY 05230, relative to all other CMs, require a change 659 of the photodissociation regime across the S condensation window (section 5.4). This is also consistent 660 with the reversal of the $\Delta^{33} S$ difference between $S^{0}$ and sulfide for these two CMs (Table 3, Fig. 8). In this 661 context, the positive $\Delta^{33} S$ values of both $S^{0}$ and sulfide in the two meteorites could be explained if these 662 phases were derived from a S reservoir that had already acquired a ${ }^{33} \mathrm{~S}$ enrichment. One could speculate 663 that the first photodissociation regime (with $\Delta^{36} \mathrm{~S} / \Delta^{33} \mathrm{~S} \sim-3$ and producing increasing $\Delta^{33} \mathrm{~S}$ values with 664 decreasing $f$ values, Fig. 8) was responsible for the ${ }^{33} \mathrm{~S}$ enrichment. In that case, the minimum $\Delta^{33} \mathrm{~S}$ value of $665 \mathrm{H}_{2} \mathrm{~S}$ at the transition between the two regimes would be constrained by the Murchison sulfide value to be at 666 least $+0.156 \pm 0.008 \%$. Figure 8 illustrates the endmember possibility where the first regime $\left(\Delta^{36} S / \Delta^{33} S \sim\right.$ -

667 3) is recorded by most of the CMs, while a second one accounts for Murchison and MCY 05230 only, with a $668 \Delta^{33} S$ fractionation of $+0.30 \%$. Considering the $\Delta^{33} S$ value of $+0.156 \pm 0.008 \%$ ond a $\Delta^{36} S / \Delta^{33} S$ of $\sim-3$, the 669 first photodissociation regime would lead the $\Delta^{36}$ S values for Murchison and MCY 05230 to be $6700.45 \pm 0.30 \%$. These two CMs however have near-zero $\Delta^{36 S}$ values with only marginal overlap with this 671 prediction (Table 3). While the uncertainties on $\Delta^{36} \mathrm{~S}$ do not allow us to completely rule out this possibility, 672 below we develop an alternative and preferred model where the isotopic signatures seen in sulfide and $\mathrm{S}^{0}$ 673 could derive from a common product that contaminated both components. We discuss this interpretation 674 later in this communication.

675

676

677

678

679

\subsubsection{0ther implications and limitations}

This model requires both $\mathrm{S}^{0}$ condensation and $\mathrm{FeS}$ formation to occur coincident with the photodissociation process. Under canonical nebula conditions, the rate of FeS formation reaction (reaction 2) would have been highest at $\sim 700 \mathrm{~K}$ (Lauretta et al., 1996,1998), but non-negligible until temperatures 
680 fell below $\sim 500 \mathrm{~K}$ (Lauretta et al., 1996,1998, Llorca and Casanova 2000). Experimental data suggest a 681 likely sublimation temperature of $\mathrm{S}^{0}$ allotropes $<370 \mathrm{~K}$, although the only available data were obtained at 1 682 bar and under $\mathrm{O}_{2}$-bearing atmospheres (Meyer 1976 and references therein). If relevant to canonical 683 nebular conditions ( $\mathrm{P} \sim 5 \times 10^{-4}$ bars, with solid grains in suspension), this is below the minimum FeS 684 formation temperature, and would argue against this working hypothesis. Note that we remain cautious 685 about ruling out such a sink for $\mathrm{S}^{0}$ because it is unclear whether photochemical production of $\mathrm{S}$ 686 intermediates that have an affinity for grain surfaces could play a role in sequestering some anomalous $\mathrm{S}$ at 687 temperatures above the sublimation temperature of $S^{0}$.

688 In this model, chondrites sample snapshots of the $\mathrm{S}^{0}$ produced by $\mathrm{H}_{2} \mathrm{~S}$ photodissociation under a 689 Rayleigh distillation process. While qualitative inferences about the process appear possible, quantitative 690 modelling of $\mathrm{S}^{0}$ abundances and isotopic compositions in CMs has not been attempted as $\Delta^{33} \mathrm{~S}$ 691 fractionations vary as a function of wavelength without producing systematic $\delta^{34} \mathrm{~S}$ variations (Chakraborty 692 et al. 2013). We illustrate a simple scenario in Figure 8 using prescribed fractionations, but do not address 693 the issue of incident wavelength or the relationship between isotopic composition and mass balance 694 because the relative rate of photodissociation to sulfidation is unknown. A point in favor of the general 695 Rayleigh model approach is that the range of $\Delta^{33} \mathrm{~S}$ anomalies, from significantly negative to positive values, 696 is consistent with a Rayleigh distillation mechanism, accompanied by inefficient accretion of $\mathrm{S}^{0}$ in CM 697 matrices as suggested by $S^{0}$ being the smallest $S$ reservoir (Table 2).

698 Model A predicts that the CM with the lowest $S^{0} \Delta^{33} S$ value will have a sulfide value that is closest to the 699 bulk composition of the gas (Fig. 8). This is LEW 87022 with a value of $-0.104 \pm 0.012 \%$ o for $S^{0}$, and $700 \quad 0.051 \pm 0.008 \%$ for sulfide (Fig. 7B). The bulk $\Delta^{33} \mathrm{~S}$ value of the nebula would be between these two values 701 (but probably closer to $-0.051 \pm 0.008 \%$, Fig. 8), i.e., clearly distinct from CDT. This would leave 702 unexplained the observation that only rare differentiated bodies show significantly negative $\Delta^{33} S$ values. 703 While the mantles of the Earth, Mars, and the Moon have near-CDT $\Delta^{33} \mathrm{~S}$ values, only subgroups of irons 704 display negative $\Delta^{33}$ S values, with an average $\Delta^{33}$ S value of $-0.027 \pm 0.008 \%$ o for IIIF (Labidi et al. 2013, 705 Franz et al. 2014, Wing and Farquhar 2015, Antonelli et al. 2014). If the $\mathrm{H}_{2} \mathrm{~S}$ in the $\mathrm{CM}$ formation region had 
706 a CDT-like S isotope composition to begin with, the model fails to explain the isotope composition of LEW 70787022.

\subsubsection{Model B-1: S-MIFs in chondrites are brought to the CMs by ice accretion}

$$
\mathrm{S}^{0}+\mathrm{H}_{2}=\mathrm{H}_{2} \mathrm{~S}(3)
$$

Under this hypothesis, the transfer of S-MIF to sulfide precursors, and ultimately to iron sulfides would be prevented. Some $\mathrm{H}_{2} \mathrm{~S}$ probably remained in the nebula after troilite formation $(\mathrm{T}<500 \mathrm{~K})$,

717 accounting for the S content of Jupiter atmosphere (Gautier et al., 2001; Ciesla, 2015). Under this 718 hypothesis, residual $\mathrm{H}_{2} \mathrm{~S}$ when subjected to UV irradiation at $\mathrm{T}<500 \mathrm{~K}$ would yield $\mathrm{S}^{0}$ that eventually would be allowed to condense. The variable $\Delta^{33} S$ values in sulfides positively correlated with the $S^{0}$ values et al., 1997; Kerridge et al., 1979; Bullock et al., 2005, 2010). and $\mathrm{S}^{0}$, under this working hypothesis. Late-accretion of nebular $\mathrm{S}^{0}$ itself by ices is seen as unlikely, as experimental data suggest that it is significantly more refractory than water under any conditions, and hence it is likely to have condensed from the gas phase well before water ice formation (Jimenez-Escobar and Munoz-Caro, 2011). As an alternative, ice accretion could deliver the remaining $\mathrm{H}_{2} \mathrm{~S}$ (freezing $\mathrm{T}<170$ K, Jimenez-Escobar and Munoz-Caro, 2011) to the CM matrices. In support of this idea, comets have been observed to host a variety of S-bearing molecules, $\mathrm{H}_{2} \mathrm{~S}$ being the dominant one, representing from 0.2 to

$7301.5 \%$ relative to $\mathrm{H}_{2} \mathrm{O}$ (Irvine et al. 2000). This $\mathrm{H}_{2} \mathrm{~S}$ gas would carry S-MIF after partial photodissociation in 731 the nebula at $\mathrm{T}<500 \mathrm{~K}$. Its release in the CM parent body during aqueous alteration would have allowed reaction 1 to occur: $\mathrm{H}_{2} \mathrm{~S}$ would have reacted with $\mathrm{Fe}^{3+}$ present in the aqueous fluids that was produced by 
734 reaction, commonly observed in the laboratory (section 5.1.3), would lead to the formation of $\mathrm{S}^{0}$ with S735 MIF

736 Observations of the least altered CM meteorite Paris supports the idea that metal would have been 737 available in the first alteration reactions (Hewins et al. 2014). Therefore, the reaction 2 could also occur on 738 the parent body. The competition between the reaction 1 and 2 would have led to the formation of $\mathrm{S}^{0}$ 739 (reaction 1) as well as a second generation of sulfides (reaction 2), both bearing identical $\Delta^{33 S}$ values. It is 740 conceivable that such a process would have resulted in the further sulfidation of troilite, leading to 741 formation of pyrrhotite (Zolensky and Thomas 1995). Regardless, because our extraction process cannot 742 distinguish between the primary sulfides (CDT-like $\Delta^{33}$ S values) and the S-MIF bearing sulfide formed by 743 reaction 2, this scenario would predict bulk sulfides $\Delta^{33} \mathrm{~S}$ values that are correlated with the values for $\mathrm{S}^{0}$, 744 which consistent with our dataset (Fig. 7b).

745

\subsubsection{Model B-2: S-S production resulting from ice irradiation}

A variant of this working hypothesis relates to UV irradiation of $\mathrm{H}_{2} \mathrm{~S}$-bearing ices. Multiple lines of experimental evidence show that various $\mathrm{S}^{0}$ species are produced by the irradiation of $\mathrm{H}_{2} \mathrm{~S}$ ices by UV (or protons) under relevant nebular conditions (Jimenez-Escobar and Munoz Caro, 2011; Jimenez-Escobar et al., 2011; Moore et al., 2007). As $\mathrm{H}_{2} \mathrm{~S}$ being in a solid (icy) state, it is not known whether its photodissociation would generate a mass-independent anomaly. We note though that the UV irradiation process of the ices in the protoplanetary nebula can be associated with warming of the ices, leading to temperatures allowing $\mathrm{H}_{2} \mathrm{~S}$ desorption (Ciesla and Sandford, 2012; Jimenez-Escobar et al., 2014). This would allow the photodissociation to have occurred under gas state, leading to production of S-MIF

755 (Chakraborty et al., 2013). If products recondensed on ice, this mechanism could constitute a possible 756 pathway for transferring S-MIFs to the CM parent body.

\subsubsection{Multiple implications and limitations of ice-based models}

759 The ices are required to have carried $\mathrm{H}_{2} \mathrm{~S}$ with heterogeneous $\mathrm{S}$ isotopic compositions to account for the 760 varying $\Delta^{36} \mathrm{~S} / \Delta^{33} \mathrm{~S}$ values observed from sample to sample (Fig. 5). This hypothesis contrasts with the 
761 suggestion that the ices carried other isotopically extreme but homogeneous components (Alexander et al. 762 2015). Naively, a correlation between water content and S content (or isotopic composition) might be 763 expected in any ice-based model, but they are not observed (Table 1 and 2). However, some water must 764 have been consumed by metal oxidation (e.g., Wilson et al., 1999; Rosenberg et al., 2001; Alexander et al., 7652010,2012 ). It is also possible that sulfides and $S^{0}$ were less mobile than water (after the ice melted), 766 and/or that $\mathrm{H}_{2} \mathrm{~S}$ was mostly in the gas phase and moved independently of the water. Thus, the observed 767 lack of a correlation between water and S is not seen as a weakness of this type of model. Model B-1 768 requires that the $\mathrm{H}_{2} \mathrm{~S}$ trapped in ices have $\mathrm{S}$ isotope compositions consistent with what is observed for $\mathrm{S}^{0}$ in 769 experiments (i.e., mainly negative $\Delta^{33} \mathrm{~S}$ values relative to CDT, Chakraborty et al., 2013), and not with the 770 residual $\mathrm{H}_{2} \mathrm{~S}$. This might be a weakness of the model, and experimental studies are needed to establish 771 whether $\mathrm{H}_{2} \mathrm{~S}$ photodissociation can lead to the formation of $\mathrm{S}^{0}$ with positive $\Delta^{33} \mathrm{~S}$ values with $\Delta^{36} \mathrm{~S} / \Delta^{33} \mathrm{~S}$ 772 values of around -3 . Under the model B-2, the ices would end up hosting both $\mathrm{H}_{2} \mathrm{~S}$ and $\mathrm{S}^{0}$ with 773 complementary S-MIFs. Both species would be released upon melting of the ice in the asteroid parent 774 body, and $\mathrm{H}_{2} \mathrm{~S}$ would be left to react with the iron species in the fluids, leading to the further production of $775 \mathrm{~S}^{0}$ and sulfides (like in model B-1). However, under the current state of knowledge, the proportion of $\mathrm{S}^{0}$ 776 provided by the ices, relative to the $\mathrm{S}^{0}$ formed by reaction 1 (whom the ice $\mathrm{H}_{2} \mathrm{~S}$ is the precursor), is 777 unknown. If the former were dominant, $\mathrm{S}^{0}$ and sulfides would have uncorrelated $\Delta^{33} \mathrm{~S}$ values. If the latter 778 were dominant, $S^{0}$ and sulfides would have negatively correlated $\Delta^{33} S$ values. In both cases, the positive 779 correlation between the $\mathrm{S}^{0}$ and $\mathrm{H}_{2} \mathrm{~S}$ values (Fig. 6b) would be left unaccounted for, constituting a significant 780 weakness of the model. However, we note that B-2 is not mutually exclusive with B-1. A combination of the 781 two models is possible, and would simply produce some of the scatter observed amongst the compositions 782 of the S-bearing components of the various CMs.

783 Finally, $\mathrm{H}_{2} \mathrm{~S}$ ice irradiation in the presence of $\mathrm{CH}_{3} \mathrm{OH}$ has been shown to lead to the formation of $\mathrm{S}$ 784 bearing organic molecules (Jimenez-Escobar et al., 2014, Mahjoub et al. 2016). This is a critical observation, 785 as if the $\mathrm{H}_{2} \mathrm{~S}$ photodissociation process occurred during the ice-warming events (associated with $\mathrm{H}_{2} \mathrm{~S}$ 786 sublimation), it could lead to the production of S-MIF (Chakraborty et al. 2013) and its transfer to organic 787 molecules. This mechanism has been explored for the production of $\mathrm{C}, \mathrm{N}$-bearing organics by UV irradiation 
of ice at high altitude in the disk, aided by vertical motions in the protoplanetary nebula (Ciesla and Sandford, 2012). Significant S-MIF has been reported for the soluble organic fraction of Murchison (with $\Delta^{33}$ S values up to $+2.00 \pm 0.05 \%$ ), but their origin remains unclear (Cooper et al., 1997). The model B-2 presented here, combined with the dynamical considerations of Ciesla and Sandford (2012), possibly 792 accounts for these signatures.

\subsection{What are the next steps to test the models?}

While both models explored here can explain the general features of $\mathrm{S}$ isotopic heterogeneity in the CMs, they also both have significant limitations. To test these models, experimental studies are needed to are needed to constrain the condensation behaviour of $\mathrm{S}^{0}$ under canonical nebular conditions, with and without UV light. It is also essential to constrain the reaction rate law of reaction 3 , to establish whether $\mathrm{S}^{0}$ in the vapor phase would back react to $\mathrm{H}_{2} \mathrm{~S}$. If not, MIF-bearing sulfide could be directly condensed, even in model B-1, without having the formation of late MIF-carrying sulfides as a requirement. Model B-1, in turn, can be tested through studies of the $\mathrm{S}^{0}$ chemistry in an aqueous environment and in the presence of $\mathrm{H}_{2}$. The rates of reactions 1 and 2 under the relevant conditions need to be explored in future studies to test our

804 hypothesis. Finally, to constrain the model B-2, it will be critical to address the possibility that $\mathrm{H}_{2} \mathrm{~S}$ ice 805 irradiation by UV (or protons), described to produce $\mathrm{S}^{0}$ and S-bearing organics (Jimenez-Escobar and Munoz Caro 2011, Jimenez-Escobar et al. 2014, Moore et al. 2007, Mahjoub et al. 2016), is associated with S-MIF production.

808 In general, if the anomalous S isotopic compositions in CMs are linked with the accreted ices, 809 predictions can be made for other types of chondrites: The various chondrites parent bodies did not 810 accrete similar amounts of ice (e.g., Clayton and Mayeda, 1999; Alexander et al., 2012). Consequently, they 811 would in principle have distinct S-MIF signatures. For example, COs are similar to CMs in terms of bulk 812 chemical composition (Wasson, 1988), although they accreted significantly less matrix (Krot et al., 2009 813 and references therein). The CO parent body, therefore, presumably accreted significantly less ice than the 814 CMs (Clayton and Mayeda, 1999). If the CMs S isotopic signature is linked with ice accretion only, COs 815 would be predicted to host mass dependant S isotopic signatures. 
816 Finally, we also treated all our data as if the CMs are pristine chondrites that were never subjected to 817 impact processes. These chondrites are, however, breccias (Metzler et al., 1992). It is conceivable that $\mathrm{S}^{0}$

818 and sulfides would be redistributed during the brecciation process. In that case, the isotopic relationship 819 between $\mathrm{S}^{0}$ and sulfides may have been obscured. That could explain why Banten is the only CM studied 820 here that has sulfide with $\Delta^{33} \mathrm{~S}$ depletion relative to $\mathrm{S}^{0}$ but lies on a $\Delta^{36} \mathrm{~S} / \Delta^{33} \mathrm{~S}$ of around -3 . Since varying $821 \Delta^{36} \mathrm{~S} / \Delta^{33} \mathrm{~S}$ between different CMs (Murchison and MCY 05230 versus the other CMs) require some degree 822 of heterogeneous accretion of MIF-bearing sulfides and $S^{0}$, an alternative is that a similar process 823 heterogeneously accreted S-bearing phases to Banten.

\subsection{Implications for sulfur chemistry in the early solar system}

826 A striking feature of the meteoritic sulfur isotope record is the lack of large mass-dependent and mass827 independent isotopic signatures, such as those seen for other elements that partitioned into multiple gas 828 phase molecular species. Spectroscopic observations of multiple S-bearing molecules in molecular clouds 829 (e.g., $\mathrm{H}_{2} \mathrm{~S}, \mathrm{OCS}, \mathrm{SO}_{2}$, $\mathrm{HSCN}, \mathrm{CH}_{2} \mathrm{~S}, \mathrm{CH}_{3} \mathrm{~S}, \mathrm{C}_{2} \mathrm{H}_{5} \mathrm{~S}, \mathrm{~S}_{3}, \mathrm{C}_{2} \mathrm{~S}$, SO, HSCS+, and $\mathrm{SO}^{+}$: Müller et al., 2005) suggest a rich 830 photochemistry and ion-molecule chemistry for S in the gas phase. As has already been discussed above, 831 photochemical reactions can produce significant S-MIF (up to $+1.5 \%$ ). The occurrence of significantly 832 smaller S-MIF in chondrites (this study) and achondrites (Farquhar et al., 2000a, Rai et al., 2005; Rai and 833 Thiemens, 2007, Antonelli et al., 2014) suggests that S may have been homogenized to a significant extent 834 in the nebula. The MIF-S signatures in achondrites have been interpreted to reflect such a processing in 835 high-temperature nebular environments, near the Sun (Rai et al., 2005; Rai and Thiemens, 2007; 836 Chakraborty et al., 2013; Antonelli et al., 2014).

837 The standard model to explain S-MIF in achondrites is based on the observation of a $+0.161 \pm 0.012 \%$ o $838 \Delta^{33}$ S values in Norton County oldhamites (Rai et al., 2005). The assertion is that sulfide such as CaS could be 839 a refractory carrier of isotope anomalies to asteroids (Rai et al., 2005). If the isotopic composition of 840 oldhamite records photo-processed $\mathrm{H}_{2} \mathrm{~S}$ (Rai et al., 2005), the production of complementary $\mathrm{S}^{0}$ carrying 841 negative $\Delta^{33} \mathrm{~S}$ values was required by this observation. This $\mathrm{S}^{0}$ must also have escaped condensation 842 consistent with its low condensation temperature, and reaction with $\mathrm{H}_{2}$ back to $\mathrm{H}_{2} \mathrm{~S}$ (i.e., reaction 3) as it 
843 would otherwise lead to the preservation of a CDT-like $\Delta^{33} \mathrm{~S}$ value in the bulk $\mathrm{H}_{2} \mathrm{~S}$ and in the condensed

844 sulfides. Later on, the CaS grains could have been transported to various nebular regions where they would 845 have been incorporated into meteorite parent bodies, possibly through X-winds (Rai et al., 2005). $\mathrm{H}_{2} \mathrm{~S}$

846 photodissociation cannot occur at the nebular midplane as it is where the optical density is the highest 847 (Ciesla, 2010). It would rather occur close to the sun, where optical density allows UV light to interact with 848 gas phases (Rai et al., 2005; Antonelli et al., 2014). Note that in addition, the reduced conditions closer to 849 the sun (produced by $\mathrm{H}_{2} \mathrm{O}$ depletion) are required by the occurrence of refractory $\mathrm{Ca}, \mathrm{Mg}$-sulfides as 850 canonical C/O nebular conditions would otherwise bind calcium and magnesium to oxygen, not sulfur 851 (Lodders, 2003).

852 Further complications arising with this model are revealed by examination of the oldhamite data from 853 aubrites. Rai et al. (2005) and Defouilloy et al. (2016) report highly variable $\Delta^{33}$ S values for oldhamite 854 separates coming from the Norton County aubrite. In addition, the incorporation of CaS in the aubrite 855 parent body must have occurred late, likely after the aubrite asteroid differentiation, so that anomalous $856 \Delta^{33} \mathrm{~S}$ values relative to bulk sulfur could be preserved in refractory sulfides (and not mixed during 857 magmatic processes). This has chronological implications on how the disk produces and distributes early858 condensed refractory sulfides to asteroids.

859 Refractory sulfides do not contribute to our extracted S (section 3.1). Only the Sutter's Mill CM, that also 860 displays evidence for mixing with enstatite chondrite during brecciation, have been reported to contain 861 oldhamites (Zolensky et al. 2014). CMs only contain Fe-(Ni-)sulfides (and tochilinites), produced by the 862 parent body processing of Fe-sulfides; Fe-sulfides being the principal sulfide phase predicted to form under 863 canonical C/O conditions (Zolensky and Thomas, 1995; Rubin et al., 2007, Lodders, 2003). The direct 864 observation of $\mathrm{S}^{0}$ carrying most of the $\Delta^{33} \mathrm{~S}$ variability, and of sulfide carrying complementary $\Delta^{33} \mathrm{~S}$ values, 865 shows that the condensation of refractory $\mathrm{CaS}$ is not required to preserve non-CDT $\Delta^{33} \mathrm{~S}$ values. In that case, 866 the $\mathrm{H}_{2} \mathrm{~S}$ photodissociation could occur at the surface of the disk, and provide another way to produce S-MIF. 867 Vertical motion is a natural consequence of realistic disk viscosities (Ciesla, 2010; Ciesla and Sandford, 868 2012). Over timescales of $<10^{5}$ years, many particles would have experienced significant UV irradiation 869 simply through vertical motions that transported matter from the midplane to high altitudes in the disk 
870 (Ciesla, 2010; Ciesla and Sandford, 2012). We thus suggest the surface of the disk as a viable location for $871 \mathrm{H}_{2} \mathrm{~S}$ photodissociation recorded in CMs.

872 Our observations do not negate the validity of the refractory sulfide model, as these phases are directly 873 observed to carry S-MIF in the aubrite meteoritical record (Rai et al., 2005; DeFouilloy et al., 2016). Rather, 874 our dataset establishes the fact that at least two S-MIF producing process were operating in the solar 875 nebula, one producing the refractory sulfides observed in aubrites, and one producing the variations 876 observed in CMs. The expansion of the refractory sulfide model to iron meteorites (Antonelli et al., 2014) 877 may be valid, but our observation offers an alternative mechanism for S photochemistry that can be 878 achieved under canonical C/O conditions, and possibly at any heliocentric distance provided a mechanism 879 exists for preserving and transferring the S-MIF to planetesimals.

$880 \quad \mathrm{~A} \mathrm{H}_{2} \mathrm{~S}$ photodissociation mechanism, similar to the ones described in our model A does not require the 881 involvement of ice accretion (or any subsequent parent body processes) to transfer S-MIF to asteroids, and 882 could then be extended to meteorite precursors formed in the inner region of the solar system like iron meteorites (Bottke et al., 2006). This scenario would lead to the formation of variable $\Delta^{33} S$ values for $S^{0}$ and FeS. Any deposited $\mathrm{S}^{0}$ is likely to have experienced reduction to sulfide during differentiation of the parent bodies of meteorites; this is because parent bodies of all known achondrites and iron meteorites have $f_{2}$ 886 only allowing $\mathrm{S}^{2-}$ as the stable S-bearing phase (e.g., Metrich et al. 2009). Thus, the heterogeneous 887 distribution of the photodissociation products and residues (like those observed amongst the CMs) could easily lead to the $\sim 0.05 \% 0 \Delta^{33} \mathrm{~S}$ variations observed in the achondrites and irons (Farquhar et al., 2000a; Antonelli et al., 2014). This process could also be recorded in the chondrule precursors of ordinary 890 chondrite, accounting for the S-MIF observed in some of these objects (Rai and Thiemens, 2007). In 891 addition to relaxing the requirement of a reduced, hot inner nebula for the production and sequestration of 892 S-MIF in the meteorite record, this model eliminates the need for the redistribution of grains formed near 893 the proto-Sun to the achondrite-forming region. Importantly, this removes any requirement for the X-wind 894 model or other disk winds to explain the occurrence of S-MIF in meteorites that form in distal cooler 895 regions of the nebula.

896 The ice-based models, in turn, could not be expanded to iron meteorite, as these bodies were likely 897 formed too close to the sun to accrete significant amount of ices (Bottke et al. 2006). However, the potential 
of S-MIF production during ice transport (by UV irradiation of the ices: Jimenez-Escobar and Munoz Caro, 2011; Ciesla and Sandford, 2012) could explain the S-MIF observed in the only study of the organic phases

900 of the Murchison meteorite (Cooper et al., 1997). This would relax the hypothesis that interstellar S was

901 involved in meteoritical organic matter (Cooper et al., 1997), and support the idea that UV processing of 902 ices before their accretion to asteroids can be associated with at least a portion of the organic matter 903 present in chondrites (Ciesla and Sandford, 2012).

904

905

906 sulfide, sulfate and $\mathrm{S}^{0}$ in $13 \mathrm{CM}$ carbonaceous chondrites. Our samples include 4 falls and 9 finds, and display an average $S$ content of $2.11 \pm 0.39$ wt.\% S $(1 \sigma)$. The recovered sulfate, $S^{0}$ and sulfide contents represent $25 \pm 12 \%, 10 \pm 7 \%$ and $65 \pm 15 \%$ of the bulk S, respectively (all $1 \sigma$ ? .

910 We report ranges of S-MIFs in carbonaceous chondrites that are larger than previously observed. The 911 largest variations are captured by $S^{0}$, with $\Delta^{33} S$ values ranging between $-0.104 \pm 0.012 \%$ (LEW 87022, $2 \sigma$ ) 912 and $+0.256 \pm 0.018 \%$ ( $M C Y 05230,2 \sigma$ ). The $\Delta^{36} \mathrm{~S} / \Delta^{33} \mathrm{~S}$ values of $\mathrm{S}^{0}$ are on average $-3.1 \pm 1.0(2 \sigma)$. When 913 sulfides and sulfates are taken into account, the average $\Delta^{36} \mathrm{~S} / \Delta^{33} \mathrm{~S}$ value is $-3.9 \pm 2.2(2 \sigma)$. The only 914 exceptions are MCY 05230 and one of two Murchison samples that have $\Delta^{36} \mathrm{~S} / \Delta^{33} \mathrm{~S}$ values of $+1.3 \pm 0.1$ and

$915+0.9 \pm 0.1$, respectively, for their $\mathrm{S}^{0}$ (note that their sulfides and sulfates display similar positive $\Delta^{36} \mathrm{~S} / \Delta^{33} \mathrm{~S}$ 916 values as well).

$917 \quad$ Non-zero $\Delta^{33} \mathrm{~S}$ values are also preserved in sulfide and sulfate, and are correlated with $\mathrm{S}^{0}$ values. The 918 observed trends suggest a genetic relationship between the extracted S-bearing phases. For sulfate, the $919 \Delta^{33} S$ trend with $S^{0}$ values has a slope of $0.86 \pm 0.24(2 \sigma)$. This slope is consistent with sulfate was produced 920 by $S^{0}$ oxidation, not sulfide oxidation, during parent body processing. For sulfide, the slope of the $\Delta^{33} S$ trend 921 with $S^{0}$ is $0.56 \pm 0.20(2 \sigma)$. This suggests that the genetic link between $\mathrm{S}^{0}$ and sulfide is more complex.

$922 \quad \mathrm{H}_{2} \mathrm{~S}$ photodissociation experiments with UV light (wavelength $<150 \mathrm{~nm}$ ) have previously demonstrated 923 the formation of MIF-bearing $\mathrm{S}^{0}$. The sign of our $\Delta^{33} \mathrm{~S}$ values, and the $\Delta^{36} \mathrm{~S} / \Delta^{33} \mathrm{~S}$ values of our S-bearing 924 extracts are consistent with the experimental data. We have explored two types of models, both requiring 
$925 \mathrm{H}_{2} \mathrm{~S}$ photodissociation, to explain our data. Although imperfect, these models explain the major features of 926 the CM S isotope compositions, and can be tested experimentally in future studies. In model A, sulfides and $927 \quad \mathrm{~S}^{0}$ are both condensates produced after incomplete $\mathrm{H}_{2} \mathrm{~S}$ photodissociation. A distillation process during 928 photodissociation would account for the sign and range of the S-MIF observed in $\mathrm{S}^{0}$ and sulfides. This 929 mechanism requires that the product of the photodissociation $\left(\mathrm{S}^{0}\right)$ is removed from the system via 930 condensation immediately after its production. It also requires the troilite formation occurs 931 contemporaneously with $\mathrm{S}^{0}$ condensation. The well-documented rate for metal sulfidation would indicate a 932 temperature window for troilite formation between $500 \mathrm{~K}$ and $700 \mathrm{~K}$. Although poorly known, the $\mathrm{S}^{0}$ 933 condensation temperature inferred from the literature seems too low to allow such process to occur.

934 In model B-1, $\mathrm{H}_{2} \mathrm{~S}$ experienced partial photodissociation at $\mathrm{T}<500 \mathrm{~K}$. Some of this $\mathrm{H}_{2} \mathrm{~S}$ would be trapped 935 in ices that were later accreted, along with the condensed $\mathrm{S}^{0}$, by the CMs in their matrices. Melting of the ice 936 in the CM parent body would have released this $\mathrm{H}_{2} \mathrm{~S}$, enabling it to react with iron in metal and in solution, 937 leading to the formation of a second generation of sulfides, as well as $\mathrm{S}^{0}$. These late phases would carry the S-MIF presently observed. This model requires ices to accrete $\mathrm{H}_{2} \mathrm{~S}$ with heterogeneous $\mathrm{S}$ isotopic compositions. A model B-2 includes the possibility of further photo-processing of $\mathrm{H}_{2} \mathrm{~S}$ in the ices prior to 940 accretion by the CM parent body.

941 Our observations offer a mechanism for S photochemistry that can be achieved under canonical C/O conditions, allowing revisiting the origin of S-MIF in other meteorites and relaxing the need for invoking

943 the X-wind model. $\mathrm{A}_{2} \mathrm{~S}$ photodissociation mechanism, similar to the ones recorded in $\mathrm{CMs}$, would lead to 944 the formation of variable $\Delta^{33} \mathrm{~S}$ values in condensed phases, and their heterogeneous distribution could 945 easily lead to the small $\Delta^{33} \mathrm{~S}$ variations observed in other meteorite groups, including non-chondrites.

\section{Acknowledgements}

949 This work was supported by a Carnegie (Geophysical Laboratory) postdoctoral fellowship granted to 950 JL. JF acknowledges the NASA Cosmochemistry grant NNX13AL13G. CA was also partially supported by 951 NASA Cosmochemistry grant NNX14AJ54G. For generously supplying the samples, the authors would like 952 to thank: the British National History Museum, the members of the Meteorite Working Group, Cecilia 
953 Satterwhite and Kevin Righter (NASA, Johnson Space Center), Tim McCoy, Cari Corrigan and Linda

954 Welzenbach (Smithsonian Institute), and Laurence Garvie (Arizona State University). US Antarctic

955 meteorite samples are recovered by the Antarctic Search for Meteorites (ANSMET) program, which has

956 been funded by NSF and NASA, and characterized and curated by the Department of Mineral Sciences of the

957 Smithsonian Institution and Astromaterials Curation Office at NASA Johnson Space Center. We thank Munir

958 Humayun and three anonymous reviewers for comments that greatly improved this study. We also thank

959 James Dottin and Michael Antonelli for comments on the manuscript. We thank Joost Hoek, Doug Rumble,

960 Anat Shahar, Alan Boss and George Cody for technical help and discussions throughout this study.

961

962

963

964

965

966

967

968

969

970

971

972

973

974

975

976

977

978

979

980

981

982

983

984

985

986

987

988

989

990

991

992

993

994

995

996

997

998

999

\section{References}

Alexander, C. M. O'D., Fogel, M., Yabuta, H. and Cody, G. D. (2007). The origin and evolution of chondrites recorded in the elemental and isotopic compositions of their macromolecular organic matter. Geochim. Cosmochim. Acta, 71, 4380-4403.

Alexander, C. M. O’D., Newsome, S. D., Fogel, M. L., Nittler, L. R., Busemann, H. and Cody, G. D. (2010). Deuterium enrichments in chondritic macromolecular material-Implications for the origin and evolution of organics, water and asteroids. Geochim. Cosmochim. Acta, 74, 4417-4437.

Alexander, C. M. O’D., Bowden, R., Fogel, M. L., Howard, K. T., Herd, C. D. K. and Nittler, L. R. (2012). The provenances of asteroids, and their contributions to the volatile inventories of the terrestrial planets. Science, 337, 721-723.

Alexander, C. M. O'D., Howard, K. T., Bowden, R. and Fogel, M. L. (2013). The classification of CM and CR chondrites using bulk H, C and N abundances and isotopic compositions. Geochim. Cosmochim. Acta, 123, 244-260.

Alexander, C. M. O'D., Bowden, R., Fogel, M. L. and Howard, K. T. (2015). Carbonate abundances and isotopic compositions in chondrites. Meteorit. Planet. Sci. 50, 810-833.

Airieau, S. A., Farquhar, J., Thiemens, M. H., Leshin, L. A., Bao, H. and Young, E. (2005). Planetesimal sulfate and aqueous alteration in $\mathrm{CM}$ and $\mathrm{Cl}$ carbonaceous chondrites. Geochim. Cosmochim. Acta, 69, 4167-4172.

Antonelli, M. A., Kim, S. T., Peters, M., Labidi, J., Cartigny, P., Walker, R. J., Lyons, J. R., Hoek, J. and Farquhar, J. (2014). Early inner solar system origin for anomalous sulfur isotopes in differentiated protoplanets. Proc. Natl. Acad. Sci., 111, 17749-17754.

Bains-Sahota, S. K. and Thiemens, M. H. (1988). Fluorination of $\mathrm{SF}_{4}, \mathrm{SF}_{5} \mathrm{Cl}$, and $\mathrm{S}_{2} \mathrm{~F}_{10}$ to $\mathrm{SF}_{6}$ for mass spectrometric isotope ratio analysis. Anal. Chem, 60, 1084-1086.

Baroni, M., Thiemens, M. H., Delmas, R. J. and Savarino, J. (2007). Mass-independent sulfur isotopic compositions in stratospheric volcanic eruptions. Science, 315, 84-87.

Beck, P., De Andrade, V., Orthous-Daunay, F. R., Veronesi, G., Cotte, M., Quirico, E. and Schmitt, B. (2012). The redox state of iron in the matrix of $\mathrm{Cl}, \mathrm{CM}$ and metamorphosed $\mathrm{CM}$ chondrites by XANES spectroscopy. Geochim. Cosmochim. Acta, 99, 305-316.

Benedix, G. K., Russell, S. S., Forman, L. V., Bevan, A. W. R. and Bland, P. A. (2015). A new unequilibrated chondrite lithology discovered in the Murchison CM2 meteorite. In Lunar Planet. Sci. XLVI. Lunar Planet. Inst., Houston \#1143(abstr.).

Bland, P. A., Jackson, M. D., Coker, R. F., Cohen, B. A., Webber, J. B. W., Lee, M. R., Duffy, C. M., Chater, R. J., Ardakani M. G., McPhail D. S., McComb, D. W. and Benedix G. K., (2009). Why aqueous alteration in asteroids was isochemical: High porosity $\neq$ high permeability. Earth Planet. Sci. Lett., 287, 559-568.

Bottke, W. F., Nesvorný, D., Grimm, R. E., Morbidelli, A. and O'Brien, D. P. (2006). Iron meteorites as remnants of planetesimals formed in the terrestrial planet region. Nature, 439, 821-824.

Boulegue, J. (1978). Solubility of elemental sulfur in water at 298 K. Phosphorus Sulfur Relat. Element, 5, $127-128$. 
1000

1001

1002

1003

1004

1005

1006

1007

1008

1009

1010

1011

1012

1013

1014

1015

1016

1017

1018

1019

1020

1021

1022

1023

1024

1025

1026

1027

1028

1029

1030

1031

1032

1033

1034

1035

1036

1037

1038

1039

1040

1041

1042

1043

1044

1045

1046

1047

1048

1049

1050

1051

1052

1053

1054

1055

1056

1057

1058

1059

1060
Bullock, E. S., Gounelle, M., Lauretta, D. S., Grady, M. M. and Russell, S. S. (2005). Mineralogy and texture of Fe$\mathrm{Ni}$ sulfides in $\mathrm{Cl} 1$ chondrites: Clues to the extent of aqueous alteration on the Cl1 parent body. Geochim. Cosmochim. Acta, 69, 2687-2700.

Bullock, E. S., McKeegan, K. D., Gounelle, M., Grady, M. M. and Russell, S. S. (2010). Sulfur isotopic composition of Fe- Ni sulfide grains in $\mathrm{Cl}$ and $\mathrm{CM}$ carbonaceous chondrites. Meteorit. Planet. Sci., 45, 885-898.

Burgess, R., Wright, I. P. and Pillinger, C. T. (1991). Determination of sulphur-bearing components in C1 and C2 carbonaceous chondrites by stepped combustion. Meteoritics, 26, 55-64.

Canfield, D. E., Raiswell, R., Westrich, J. T., Reaves, C. M. and Berner, R. A. (1986). The use of chromium reduction in the analysis of reduced inorganic sulfur in sediments and shales. Chem. Geol., 54, 149-155.

Chakraborty, S., Jackson, T. L., Ahmed, M. and Thiemens, M. H. (2013). Sulfur isotopic fractionation in vacuum UV photodissociation of hydrogen sulfide and its potential relevance to meteorite analysis. Proc. Natl. Acad. Sci., 110, $17650-17655$.

Ciesla, F. J. (2010). Residence times of particles in diffusive protoplanetary disk environments. I. Vertical motions. Astrophys. J., 723, 514.

Ciesla, F. J. and Sandford, S. A. (2012). Organic synthesis via irradiation and warming of ice grains in the solar nebula. Science, 336, 452-454.

Ciesla, F. J. (2015). Sulfidization of iron in the dynamic solar nebula and implications for planetary compositions. Astrophys. J. Letters, 800, L6.

Clayton, R. N. and Mayeda, T. K. (1996). Oxygen isotope studies of achondrites. Geochim. Cosmochim. Acta, 60, 1999-2017.

Cooper, G. W., Thiemens, M. H., Jackson, T. L. and Chang, S. (1997). Sulfur and hydrogen isotope anomalies in meteorite sulfonic acids. Science, 277, 1072-1074.

Craig, H. (1957). Isotopic standards for carbon and oxygen and correction factors for mass-spectrometric analysis of carbon dioxide. Geochim. Cosmochim. Acta, 12, 133-149.

Davidson, J., Busemann, H., Nittler, L. R., Alexander, C. M. O’D., Orthous-Daunay, F. R., Franchi, I. A. and Hoppe, P. (2014). Abundances of presolar silicon carbide grains in primitive meteorites determined by NanoSIMS. Geochim. Cosmochim. Acta, 139, 248-266.

Defouilloy, C., Cartigny, P., Assayag, N., Moynier, F. and Barrat, J. A. (2016). High-precision sulfur isotope composition of enstatite meteorites and implications of the formation and evolution of their parent bodies. Geochim. Cosmochim. Acta, 172, 393-409.

Desch, S. J., Morris, M. A., Connolly Jr, H. C. and Boss, A. P. (2010). A critical examination of the X-wind model for chondrule and calcium-rich, aluminum-rich inclusion formation and radionuclide production. Astrophys. J., 725, 692.

Dreibus, G., Palme, H., Spettel, B., Zipfel, J. and Wänke, H. (1995). Sulfur and selenium in chondritic meteorites. Meteoritics, 30, 439-445.

Eiler, J., Cartigny, P., Hofmann, A. E. and Piasecki, A. (2013). Non-canonical mass laws in equilibrium isotopic fractionations: Evidence from the vapor pressure isotope effect of SF 6 . Geochim. Cosmochim. Acta, 107, 205219.

Farquhar, J., Jackson, T. L. and Thiemens, M. H. (2000)a. A ${ }^{33}$ S enrichment in ureilite meteorites: evidence for a nebular sulfur component. Geochim. Cosmochim. Acta, 64, 1819-1825.

Farquhar, J., Savarino, J., Jackson, T. L. and Thiemens, M. H. (2000)b. Evidence of atmospheric sulphur in the martian regolith from sulphur isotopes in meteorites. Nature, 404, 50-52.

Fitzgerald, M. J. and Jaques, A. L. (1982). Tibooburra, a new Australian meteorite find, and other carbonaceous chondrites of high petrologic grade. Meteoritics, 17, 9-26.

Fuchs, L. H., Jensen, K. J. and Olsen, E. J. (1973). Mineralogy, mineral-chemistry, and composition of the Murchison C2 meteorite. Smithson. Contrib. Earth Sci. 10, 39

Fujiya, W., Hoppe, P., Zinner, E., Pignatari, M. and Herwig, F. (2013). Evidence for Radiogenic Sulfur-32 in Type AB Presolar Silicon Carbide Grains?. Astrophys. J. Letters, 776, L29.

Sugiura, N. and Fujiya, W. (2014). Correlated accretion ages and $\varepsilon^{54} \mathrm{Cr}$ of meteorite parent bodies and the evolution of the solar nebula. Meteorit. Planet. Sci., 49, 772-787.

Franz, H. B., Kim, S. T., Farquhar, J., Day, J. M., Economos, R. C., McKeegan, K. D., Schmitt, A. K., Irving, A. J., Hoek and J., Dottin III, J. (2014). Isotopic links between atmospheric chemistry and the deep sulphur cycle on Mars. Nature, 508, 364-368.

Fry, B., Ruf, W., Gest, H. and Hayes, J. M. (1988). Sulfur isotope effects associated with oxidation of sulfide by $\mathrm{O}_{2}$ in aqueous solution. Chem. Geol., 73, 205-210.

Gao, X. and Thiemens, M. H. (1993). Isotopic composition and concentration of sulfur in carbonaceous chondrites. Geochim. Cosmochim. Acta, 57, 3159-3169.

Gautier, D., Hersant, F., Mousis, O. and Lunine, J. I. (2001). Enrichments in volatiles in Jupiter: a new interpretation of the Galileo measurements. Astrophys. J. Lett., 550, L227-L230.

Gounelle, M. and Zolensky, M. E. (2001). A terrestrial origin for sulfate veins in Cl1 chondrites. Meteorit. Planet. Sci., 36: 1321-1329. 
1061

1062

1063

1064

1065

1066

1067

1068

1069

1070

1071

1072

1073

1074

1075

1076

1077

1078

1079

1080

1081

1082

1083

1084

1085

1086

1087

1088

1089

1090

1091

1092

1093

1094

1095

1096

1097

1098

1099

1100

1101

1102

1103

1104

1105

1106

1107

1108

1109

1110

1111

1112

1113

1114

1115

1116

1117

1118

1119

1120

1121

1122
Graham, A. L., Yanai, K., Kojima, H. and Ikadai, S. (1985). Yamato 81020 and Yamato 82042; two new carbonaceous chondrites from Antarctica. Meteoritics, 20, 654.

Hewins, R. H., Bourot-Denise, M., Zanda, B., Leroux, H., Barrat, J. A., Humayun, M., Gopel, C., Greenwood., R. C., Franchi, I. A., Pont, S., Lorand, J. P., Cournede, C., Gattacceca, J., Rochette, P., Kuga, M., Marrocchi, Y., and Marty, B., (2014). The Paris meteorite, the least altered CM chondrite so far. Geochim. Cosmochim. Acta, 124, 190-222.

Hoppe, P., Fujiya, W. and Zinner, E. (2012). Sulfur molecule chemistry in supernova ejecta recorded by silicon carbide stardust. Astrophys. J. Lett., 745, L26.

Howard, K. T., Alexander, C. M. O'D., Schrader, D. L. and Dyl, K. A. (2015). Classification of hydrous meteorites (CR, CM and $\mathrm{C} 2$ ungrouped) by phyllosilicate fraction: PSD-XRD modal mineralogy and planetesimal environments. Geochim. Cosmochim. Acta, 149, 206-222.

Hulston, J. R. and Thode, H. G. (1965). Variations in the $S^{33}, S^{34}$, and $S^{36}$ contents of meteorites and their relation to chemical and nuclear effects. J., Geophys. Res., 70, 3475-3484.

Jarosewich, E. (1971). Chemical analysis of the Murchison meteorite. Meteoritics, 6, 49-52.

Kamyshny, A., Borkenstein, C. G. and Ferdelman, T. G. (2009). Protocol for Quantitative Detection of Elemental Sulfur and Polysulfide Zero-Valent Sulfur Distribution in Natural Aquatic Samples. Geostand. Geoanal. Res., 33, 415-435.

Kaplan, I. R. and Hulston, J. R. (1966). The isotopic abundance and content of sulfur in meteorites. Geochim. Cosmochim. Acta, 30, 479-496.

Keller-Rudek, H., Moortgat, G. K., Sander, R., and Sörensen, R. (2013) The MPI-Mainz UV/VIS spectral atlas of gaseous molecules of atmospheric interest. Earth Syst. Sci. Data, 5, 365-373

Kerridge, J. F., Macdougall, J. D. and Marti, K. (1979). Clues to the origin of sulfide minerals in Cl chondrites. Earth Planet. Sci. Lett., 43, 359-367.

Kinnunen, K. A. and Saikkonen, R. (1983). Kivesvaara C2 chondrite: Silicate petrography and chemical composition. Bull. Geol. Soc. Finland 55, 35-49.

Krot, A. N., Amelin, Y., Bland, P., Ciesla, F. J., Connelly, J., Davis, A. M., Huss, G. R., Hutcheon, I. D., Makide, K., Nagashima, K., Nyquist, L. E., Russell, S. S., Scott, E. R. D, Thrane, K., Yurimoto, H. and Yin Q. Z., (2009). Origin and chronology of chondritic components: A review. Geochim. Cosmochim. Acta, 73, 4963-4997.

Labidi, J., Cartigny, P., Birck, J. L., Assayag, N. and Bourrand, J. J. (2012). Determination of multiple sulfur isotopes in glasses: A reappraisal of the MORB $\delta^{34} S$. Chem. Geol., 334, 189-198.

Labidi, J., Cartigny, P. and Moreira, M. (2013). Non-chondritic sulphur isotope composition of the terrestrial mantle. Nature, 501, 208-211.

Lauretta, D. S., Kremser, D. T. and Fegley Jr, B. (1996). The rate of iron sulfide formation in the solar nebula. Icarus, 122, 288-315.

Lauretta, D. S., Lodders, K. and Fegley, B. (1997). Experimental simulations of sulfide formation in the solar nebula. Science, 277, 358-360.

Lewis, J. S. and Krouse, H. R. (1968). Isotopic composition of sulfur and sulfate produced by oxidation of FeS. Earth Planet. Sci. Lett., 5, 425-428.

Lodders, K. (2003). Solar system abundances and condensation temperatures of the elements. Astrophys. J., 591, 1220.

Ma, C., Beckett, J. R. and Rossman, G. R. (2010). Discovery of a new chromium sulfide mineral, $\mathrm{Cr}_{5} \mathrm{~S}_{6}$ in Murchison. Meteorit. Planet. Sci., 45, A124.

Mahjoub, A., Poston, M. J., Hand, K. P., Brown, M. E., Hodyss, R., Blacksberg, J., Eiler, J. M., Carlon R. W., Ehlmann B. L., and Choukroun, M. (2016). Electron Irradiation and Thermal Processing of Mixed-ices of Potential Relevance to Jupiter Trojan Asteroids. Astrophys. J., 820, 141.

Marrocchi, Y. and Libourel, G. (2013). Sulfur and sulfides in chondrules. Geochim. Cosmochim. Acta, 119, 117136.

Mason, B. (1963). The carbonaceous chondrites. Space Sci. Rev., 1, 621-646.

Matsuhisa, Y., Goldsmith, J. R. and Clayton, R. N. (1978). Mechanisms of hydrothermal crystallization of quartz at $250^{\circ} \mathrm{C}$ and $15 \mathrm{kbar}$. Geochim. Cosmochim. Acta, 42, 173-182.

Mayer, B. and Krouse, H. R. (2004). Procedures for sulfur isotope abundance studies. In Handbook of stable isotope analytical techniques, vol. 1 (ed. P. A. de Groot). Elsevier, Amsterdam, pp. 538-603 (Chapter 26).

McEwincg, C. E., Thode, H. G. and Rees, C. E. (1980). Sulphur isotope effects in the dissociation and evaporation of troilite: A possible mechanism for ${ }^{34} \mathrm{~S}$ enrichment in lunar soils. Geochim. Cosmochim. Acta, 44, 565-571.

Métrich, N., Berry, A. J., O'Neill, H. S. C. and Susini, J. (2009). The oxidation state of sulfur in synthetic and natural glasses determined by X-ray absorption spectroscopy. Geochim. Cosmochim. Acta, 73, 2382-2399.

Metzler, K., Bischoff, A. and Stöffler, D. (1992). Accretionary dust mantles in CM chondrites: Evidence for solar nebula processes. Geochim. Cosmochim. Acta, 56, 2873-2897.

Monster, J., Anders, E. and Thode, H. G. (1965). ${ }^{34} \mathrm{~S} /{ }^{32} \mathrm{~S}$ ratios for the different forms of sulphur in the Orgueil meteorite and their mode of formation. Geochim. Cosmochim. Acta, 29, 773-779.

Müller, H. S., Schlöder, F., Stutzki, J. and Winnewisser, G. (2005). The Cologne Database for Molecular Spectroscopy, CDMS: a useful tool for astronomers and spectroscopists. J. Mol. Struct., 742, 215-227. 
1123

1124

1125

1126

1127

1128

1129

1130

1131

1132

1133

1134

1135

1136

1137

1138

1139

1140

1141

1142

1143

1144

1145

1146

1147

1148

1149

1150

1151

1152

1153

1154

1155

1156

1157

1158

1159

1160

1161

1162

1163

1164

1165

1166

1167

1168

1169

1170

1171

1172

1173

1174

1175

1176

1177

1178

1179
Ono, S., Wing, B. Rumble, D. and Farquhar, J. (2006). High precision analysis of all four stable isotopes of sulfur $\left({ }^{32} \mathrm{~S},{ }^{33} \mathrm{~S},{ }^{34} \mathrm{~S}\right.$ and $\left.{ }^{36} \mathrm{~S}\right)$ at nanomole levels using a laser fluorination isotope-ratio-monitoring gas chromatography-mass spectrometry. Chem. Geol., 225, 30-39.

Pasek, M. A., Milsom, J. A., Ciesla, F. J., Lauretta, D. S., Sharp, C. M. and Lunine, J. I. (2005). Sulfur chemistry with time-varying oxygen abundance during Solar System formation. Icarus, 175, 1-14.

Pignatari, M., Zinner, E., Bertolli, M. G., Trappitsch, R., Hoppe, P., Rauscher, T., Fryer, C., Herwig, F., Hirschi, R., Timmes, F. X., and Thielemann, F. K. (2013). Silicon carbide grains of type c provide evidence for the production of the unstable isotope ${ }^{32} \mathrm{Si}$ in supernovae. Astrophys. J. Lett., 771, L7.

Pruden, G. and Bloomfield, C. (1968). The determination of iron (II) sulphide in soil in the presence of iron (III) oxide. Analyst, 93, 532-534.

Rai, V. K., Jackson, T. L. and Thiemens, M. H. (2005). Photochemical mass-independent sulfur isotopes in achondritic meteorites. Science, 309, 1062-1065.

Rai, V. K. and Thiemens, M. H. (2007). Mass independently fractionated sulfur components in chondrites. Geochim. Cosmochim. Acta, 71, 1341-1354.

Rees, C. E. and Thode, H. G. (1977). A ${ }^{33} \mathrm{~S}$ anomaly in the Allende meteorite. Geochim. Cosmochim. Acta, 41, 1679-1682.

Rice, C. A., Tuttle, M. L. and Reynolds, R. L. (1993). The analysis of forms of sulfur in ancient sediments and sedimentary rocks: comments and cautions. Chem. Geol., 107, 83-95.

Rosenberg, N. D., Browning, L. and Bourcier, W. L. (2001). Modeling aqueous alteration of CM carbonaceous chondrites. Meteorit. Planet. Sci., 36, 239-244.

Rubin, A. E., Trigo-Rodríguez, J. M., Huber, H. and Wasson, J. T. (2007). Progressive aqueous alteration of CM carbonaceous chondrites. Geochim. Cosmochim. Acta, 71, 2361-2382.

Rumble, D., Hoering, T. C. and Palin, J. M. (1993). Preparation of $\mathrm{SF}_{6}$ for sulfur isotope analysis by laser heating sulfide minerals in the presence of $F_{2}$ gas. Geochim. Cosmochim. Acta, 57, 4499-4512.

Shu, F. H., Shang, H., Glassgold, A. E. and Lee, T. (1997). X-rays and fluctuating X-winds from protostars. Science, 277, 1475-1479.

Shu, F. H., Shang, H., Gounelle, M., Glassgold, A. E. and Lee, T. (2001). The origin of chondrules and refractory inclusions in chondritic meteorites. Astrophys. J., 548, 1029.

Simon, S. B., Sutton, S. R. and Grossman, L. (2007). Valence of titanium and vanadium in pyroxene in refractory inclusion interiors and rims. Geochim. Cosmochim. Acta, 71, 3098-3118.

Sutton, S., Cloutis, E. A. and Alexander, C. M. O'D. (2013). The Valence State of Fe and the Origin of Water in Chondrites. Lunar Planet. Sci. XLIV. Lunar Planet. Inst., Houston \#2357(abstr.).

Tachibana, S. and Huss, G. R. (2005). Sulfur isotope composition of putative primary troilite in chondrules from Bishunpur and Semarkona. Geochim. Cosmochim. Acta, 69, 3075-3097.

Thode, H. G., Monster, J. and Dunford, H. B. (1961). Sulphur isotope geochemistry. Geochim. Cosmochim. Acta, 25, 159-174.

Young, E. D., Galy, A. and Nagahara, H. (2002). Kinetic and equilibrium mass-dependent isotope fractionation laws in nature and their geochemical and cosmochemical significance. Geochim. Cosmochim. Acta, 66, 10951104.

Wing, B. A. and Farquhar, J. (2015). Sulfur isotope homogeneity of lunar mare basalts. Geochim. Cosmochim. Acta, 170, 266-280.

Wilson, L., Keil, K., Browning, L. B., Krot, A. N. and Bourcier, W. (1999). Early aqueous alteration, explosive disruption, and reprocessing of asteroids. Meteorit. Planet. Sci., 34, 541-557.

Zahnle, K. J. and Walker, J. C. (1982). The evolution of solar ultraviolet luminosity. Rev. Geophys., 20, $280-292$.

Zolensky, M. E., Bourcier, W. L. and Gooding, J. L. (1989). Aqueous alteration on the hydrous asteroids: Results of EQ3/6 computer simulations. Icarus, 78, 411-425.

Zolensky, M. E. and Thomas, K. L. (1995). Iron and iron-nickel sulfides in chondritic interplanetary dust particles. Geochim. Cosmochim. Acta, 59, 4707-4712.

Zolensky, M. E., Mittlefehldt, D. W., Lipschutz, M. E., Wang, M. S., Clayton, R. N., Mayeda, T. K., Grady, M. M., Pillinger, C. and David, B. (1997). CM chondrites exhibit the complete petrologic range from type 2 to 1. Geochim. Cosmochim. Acta, 61, 5099-5115.

Zolensky, M., Mikouchi, T., Fries, M., Bodnar, R., Jenniskens, P., Yin, Q. Z., Hagiya, K., Ohsumi, K., Komatsu, M., Colbert, M., Hanna, R., Maisano, J., Ketcham, R., Kebukawa, Y., Nakamura, T., Matsuoka, M., Sasaki, S., Tsuchiyama, A., Gounelle, M., Le, L., Martinez, J., Ross, K., And Rahman, Z. Mineralogy and petrography of C asteroid regolith: The Sutter's Mill CM meteorite. Meteorit. Planet. Sci., 49: 1997-2016. 


\section{Table 1}

A list of the studied samples, with estimated bulk $\mathrm{H}$ contents in water/OH and bulk $\delta \mathrm{D}$ composition, as reported in Alexander et al. (2013). Sample identification is given for falls. BM stands for British Museum, ASU for Arizona State University, and USNM for the Smithsonian institute. All the samples are of CM2 type except PCA 91084, which is a heated CM (Alexander et al., 2007, 2013). Two petrologic classification scales are presented here, after Alexander et al. (2013). On the first scale, the samples are distributed according to their petrologic types using a scale from 2 to 3 , based on correlations between bulk $\mathrm{H}$ isotopic compositions and the petrologic criteria of Browning et al. (1996) and Rubin et al. (2007). On the other scale, the samples are on a scale of 1 to 3 based on bulk water/OH H contents.

\section{Table 2}

The sulfur contents and speciation in the 13 studied CMs, obtained with our wet chemistry technique. Unless stated otherwise, the $\mathrm{S}$ contents were obtained by weighing of $\mathrm{Ag}_{2} \mathrm{~S}$ generated after chemical extraction of the $\mathrm{S}$ from the meteorites. The uncertainty in the abundances is $\sim 100$ ppm $(1 \sigma)$ and S content estimates for individual meteorites were rounded to the nearest 100. For $\mathrm{S}^{0}$, the abundance values obtained via High Performance Liquid Chromatography (HPLC) are also presented. The AVS, step 12 and step 13 pools refer to the fractions of extracted sulfide S using our extraction protocol. The AVS pool (for Acid Volatile Sulfur) is the $\mathrm{H}_{2} \mathrm{~S}$ generated when the CM powders were processed in hot $\mathrm{HCl}$. Step 12 and 13 reflect the collection of $\mathrm{S}^{0}$ that had been produced during the AVS step (reaction 1, see section 3.1). These three extracts were pooled by mass balance and considered to represent the bulk sulfide. See sections 3.1, 5.1.3, and Appendix A for details.

\section{Table 3}

The sulfur isotopic composition of all S extracts in the 13 studied CMs. We report $2 \sigma$ uncertainties of $0.10,0.012$ and $0.25 \%$ for $\delta^{34} S, \Delta^{33} S$ and $\Delta^{36} S$ values, respectively, for all 
measurements with a $\mathrm{SF}_{6}$ amount $>1 \mu$ mol. When $\mathrm{SF}_{6}$ amount $<1 \mu$ mol $(\mathrm{n}=4)$, we report $2 \sigma$ uncertainties of $0.10,0.018$ and $0.35 \%$ for $\delta^{34} \mathrm{~S}, \Delta^{33} \mathrm{~S}$ and $\Delta^{36} \mathrm{~S}$ values, respectively.

\section{Table 4}

The sulfur isotopic composition of all S extracts in Murchison and Cold Bokkeveld replicates. We report $2 \sigma$ uncertainties of $0.10,0.012$ and $0.25 \%$ for $\delta^{34} \mathrm{~S}, \Delta^{33} \mathrm{~S}$ and $\Delta^{36} \mathrm{~S}$ values, respectively, for all measurements. The $\delta^{34} \mathrm{~S}$ values are given versus $\mathrm{V}$-CDT. The uncertainty in the abundances is $\sim 100 \mathrm{ppm}(1 \sigma)$ and $\mathrm{S}$ content estimates for individual meteorites were rounded to the nearest 100 . 


\begin{tabular}{|c|c|c|c|c|c|c|}
\hline \multirow[b]{3}{*}{ Banten } & \multirow{3}{*}{$\begin{array}{c}\text { fall/find } \\
\text { fall }\end{array}$} & \multirow{3}{*}{$\begin{array}{l}\text { sample ID } \\
\text { USNM } 6017\end{array}$} & \multicolumn{2}{|c|}{ petrologic types } & \multicolumn{2}{|c|}{$\mathrm{H}$ content and isotope composition in bulk sample } \\
\hline & & & Based on $\mathrm{H}$ content ${ }^{\mathrm{a}}$ & based on $D^{b}$ & Water/OH H $(\text { wt\% })^{c}$ & $D(\% o)^{d}$ \\
\hline & & & 1.7 & 2.5 & 0.91 & -32.4 \\
\hline Cold Bokkeveld & fall & BM. 1727 & 1.3 & 2.2 & 1.22 & -168.3 \\
\hline Murchison & fall & BM.1988,M23 & 1.6 & 2.4 & 0.96 & -61.7 \\
\hline Nogoya & fall & ASU \#556.2 & 1.1 & 2.1 & 1.31 & -181.5 \\
\hline ALH 84029 & find & n.a. & 1.2 & 2.1 & 1.30 & -184.1 \\
\hline DOM 08003 & find & n.a. & 1.1 & 2.2 & 1.36 & -137.0 \\
\hline DOM 08013 & find & n.a. & 1.8 & 2.7 & 0.85 & 47.2 \\
\hline GRA 98074 & find & n.a. & 1.6 & 2.6 & 1.00 & -14.1 \\
\hline LEW 85312 & find & n.a. & 1.8 & 2.9 & 0.83 & 126.3 \\
\hline LEW 87022 & find & n.a. & 1.4 & 2.3 & 1.09 & -112.9 \\
\hline MCY 05230 & find & n.a. & 1.8 & 2.5 & 0.87 & -17.6 \\
\hline PCA $91084^{e}$ & find & n.a. & 1.8 & 2.4 & 0.85 & -74.6 \\
\hline TIL 91722 & find & n.a. & 1.9 & 2.7 & 0.79 & 53.7 \\
\hline
\end{tabular}

a

b

c

d

$\mathrm{e}$

petrologic type on a scale from 1 to 2 , see Alexander et al. (2013); Petrologic type $=3-\mathrm{H}_{\text {water } / \mathrm{OH}} / 0.7$ petrologic type on a scale from 2 to 3 , see Alexander et al. (2013); Petrologic type $=(D+994) / 383$ typical 1 uncertainty is $<0.03 \mathrm{wt} \%$ typical 1 uncertainty is $<10 \%$ heated CM 


\begin{tabular}{|c|c|c|c|c|c|c|c|c|c|}
\hline & mass processed (mg) & sulfate & $s^{0}$ & step 13 & step 12 & AVS & sulfide & sum & $S^{0} H P L C$ \\
\hline ALH 84029 & 206 & 4,800 & 5,500 & 8,500 & 500 & 500 & 9,500 & 19,800 & 5,800 \\
\hline Banten & 197 & 6,500 & 800 & 11,000 & 900 & 700 & 12,600 & 19,900 & 900 \\
\hline Cold Bokkeveld & $214-436^{a}$ & 5,300 & 1,300 & 12,000 & 500 & 200 & 12,700 & 19,300 & 1,100 \\
\hline DOM 08003 & 194 & 6,800 & 2,800 & 8,600 & 200 & 1,000 & 9,800 & 19,400 & 3,700 \\
\hline DOM 08013 & 192 & 6,300 & 3,100 & 17,600 & 1,000 & 2,200 & 20,800 & 30,200 & 3,200 \\
\hline GRA 98074 & 198 & 2,400 & 1,000 & 13,100 & 500 & 100 & 13,700 & 17,100 & 700 \\
\hline LEW 85312 & 198 & 6,000 & 400 & 12,700 & 700 & 1,300 & 14,700 & 21,100 & 500 \\
\hline LEW 87022 & 206 & 2,000 & 1,200 & 14,100 & 700 & 3,100 & 17,900 & 21,100 & 1,200 \\
\hline MCY 05230 & 202 & 1,100 & 1,300 & 12,300 & 200 & 3,100 & 15,600 & 18,000 & 1,300 \\
\hline Murchison & $201-202^{\mathrm{a}}$ & 5,900 & 1,600 & 7,500 & 600 & 200 & 8,300 & 15,800 & 12,300 \\
\hline Nogoya & 204 & 3,700 & 1,400 & 16,100 & 800 & 900 & 17,800 & 22,900 & 1,400 \\
\hline PCA 91084 & 211 & 13,000 & 3,200 & 10,800 & 500 & 100 & 11,400 & 27,600 & 2,100 \\
\hline TIL 91722 & 208 & 5,955 & 1,500 & 11,000 & 500 & 1,900 & 13,400 & 20,855 & 1,300 \\
\hline average & & 5,400 & 1,900 & 11, & 600 & 1,2 & 13,700 & $21,($ & 2,700 \\
\hline std dev & & 3,000 & 1,400 & 2,900 & 300 & 1,100 & 3,600 & 4,000 & 3,200 \\
\hline
\end{tabular}

all abundances are given in ppm S

a: range of values for replicates. See table $4 . S$ abundance estimates are averages of the replicates 


\begin{tabular}{|c|c|c|c|c|c|c|c|c|c|c|c|c|c|c|}
\hline \multirow{5}{*}{$s^{0}$} & & ALL 84029 & Banten & Cold Bokkeveld & DOM 08003 & DOM 08013 & GRA 98074 & LEW 85312 & LEW 87022 & MCY 05230 & Murchison & Nogoya & PCA 91084 & TIL 91722 \\
\hline & ${ }^{34} \mathrm{~S}(\%)$ V-CDT & 2.42 & 4.28 & 2.88 & 3.25 & 3.23 & 4.06 & 3.69 & 5.29 & 3.17 & 3.71 & 3.16 & -1.74 & 3.63 \\
\hline & ${ }^{34} \mathrm{~S}(\%)$ CDT & 2.32 & 4.18 & 2.78 & 3.15 & 3.13 & 3.96 & 3.60 & 5.19 & 3.07 & 3.61 & 3.06 & -1.84 & 3.53 \\
\hline & ${ }^{33 \mathrm{~S}}(\%)$ & 0.018 & -0.001 & -0.001 & $-0.034( \pm 0.018)$ & -0.085 & -0.055 & $-0.080( \pm 0.018)$ & -0.104 & 0.256 & 0.243 & -0.002 & 0.045 & 0.005 \\
\hline & ${ }^{36} 5(\%)$ & 0.00 & 0.04 & 0.09 & $0.12( \pm 0.35)$ & 0.07 & 0.23 & & 0.34 & 0.32 & 0.23 & 0.20 & -0.23 & 0.20 \\
\hline \multirow{4}{*}{$\mathrm{SO}_{4}$} & ${ }^{34}$ S (\%) V-CDT & -1.65 & -0.66 & -0.70 & -1.22 & -1.68 & -0.15 & -4.44 & 0.19 & -2.42 & -0.64 & -1.92 & 1.75 & -0.13 \\
\hline & ${ }^{34} \mathrm{~S}(\%)$ CDT & -1.75 & -0.76 & -0.80 & -1.31 & -1.78 & -0.25 & -4.54 & 0.09 & -2.52 & -0.74 & -2.02 & 1.65 & -0.23 \\
\hline & ${ }^{33} \mathrm{~S}(\%)$ & 0.058 & 0.040 & 0.023 & 0.006 & -0.036 & -0.019 & -0.023 & -0.036 & $0.143( \pm 0.018)$ & 0.263 & 0.034 & 0.016 & -0.007 \\
\hline & ${ }^{36} S(\%)$ & -0.28 & -0.23 & -0.09 & -0.21 & -0.25 & -0.03 & -0.30 & -0.14 & $0.26( \pm 0.35)$ & -0.11 & -0.20 & -0.15 & -0.33 \\
\hline \multirow{4}{*}{ Avs } & ${ }^{34} \mathrm{~S}(\%)$ V-CDT & 0.84 & 0.73 & & 0.55 & -0.38 & & 0.20 & 1.15 & 0.84 & & 1.35 & & -0.91 \\
\hline & ${ }^{34} \mathrm{~S}(\%)$ CDT & 0.75 & 0.63 & & 0.45 & -0.48 & & 0.11 & 1.05 & 0.74 & & 1.25 & & -1.00 \\
\hline & ${ }^{33} \mathrm{~S}(\%)$ & 0.027 & 0.007 & & 0.016 & 0.006 & & 0.010 & -0.022 & 0.113 & & 0.016 & & 0.033 \\
\hline & ${ }^{36} S(\%)$ & 0.25 & -0.03 & & 0.12 & -0.26 & & -0.07 & 0.18 & 0.28 & & 0.25 & & -0.56 \\
\hline \multirow{4}{*}{ step 12} & ${ }^{34}$ S (\%) V-CDT & 2.18 & 0.25 & & 4.64 & -0.03 & 0.50 & 1.07 & 1.15 & 3.90 & & 0.34 & 0.10 & 1.53 \\
\hline & ${ }^{34} \mathrm{~S}(\%)$ CDT & 2.08 & 0.15 & & 4.54 & -0.13 & 0.40 & 0.98 & 1.05 & 3.80 & & 0.24 & 0.00 & 1.43 \\
\hline & ${ }^{33} \mathrm{~S}(\%)$ & 0.046 & -0.050 & & $0.000( \pm 0.018)$ & -0.027 & -0.023 & 0.015 & -0.096 & 0.118 & & 0.033 & 0.025 & 0.016 \\
\hline & ${ }^{36} S(\%)$ & 0.80 & -0.06 & & $0.42( \pm 0.35)$ & 0.02 & -0.20 & 0.20 & 0.07 & 1.16 & & 0.23 & -0.28 & 0.00 \\
\hline \multirow{4}{*}{ step 13} & ${ }^{34}$ S (\%) V-CDT & 0.16 & -0.06 & & -0.72 & -0.50 & -0.35 & 0.20 & 0.09 & 0.21 & & 0.02 & 0.20 & -0.19 \\
\hline & ${ }^{34} \mathrm{~S}(\%)$ CDT & 0.06 & -0.16 & & -0.82 & -0.60 & -0.44 & 0.10 & -0.01 & 0.11 & & -0.08 & 0.10 & -0.29 \\
\hline & ${ }^{33} \mathrm{~S}(\%)$ & 0.047 & -0.037 & & 0.007 & 0.013 & -0.014 & -0.075 & -0.049 & 0.106 & & 0.006 & 0.015 & 0.009 \\
\hline & ${ }^{36} S(\%)$ & 0.12 & 0.07 & & 0.21 & -0.10 & -0.04 & -0.11 & -0.11 & 0.19 & & 0.14 & -0.03 & 0.00 \\
\hline \multirow{4}{*}{ bulk sulfideb } & ${ }^{34} \mathrm{~S}(\%)$ V-CDT & 0.27 & -0.04 & -0.53 & -0.60 & -0.48 & -0.31 & 0.25 & 0.15 & 0.28 & 0.24 & 0.03 & 0.19 & -0.11 \\
\hline & ${ }^{34} \mathrm{~S}(\%)$ CDT & 0.17 & -0.14 & -0.63 & -0.70 & -0.58 & -0.41 & 0.15 & 0.05 & 0.18 & 0.14 & -0.07 & 0.09 & -0.21 \\
\hline & 整s (\%०) & 0.047 & -0.038 & 0.015 & 0.007 & 0.011 & -0.014 & -0.070 & -0.051 & 0.106 & 0.156 & 0.007 & 0.015 & 0.009 \\
\hline & ${ }^{36} \mathrm{~S}(\%)$ & 0.16 & 0.06 & 0.05 & 0.22 & -0.09 & -0.04 & -0.09 & -0.10 & 0.20 & -0.01 & 0.14 & -0.04 & 0.00 \\
\hline \multirow{4}{*}{ bulk $s^{c}$} & ${ }^{34} \mathrm{~S}$ (\%) V-CDT & 0.40 & -0.06 & -0.32 & -0.09 & -0.35 & -0.04 & -1.01 & 0.43 & 0.32 & 0.24 & -0.10 & 0.70 & 0.15 \\
\hline & ${ }^{34} \mathrm{~S}(\%)$ CDT & 0.30 & -0.16 & -0.42 & -0.19 & -0.45 & -0.14 & -1.11 & 0.33 & 0.23 & 0.14 & -0.20 & 0.60 & 0.05 \\
\hline & ${ }^{33} \mathrm{~S}(\%)$ & 0.041 & -0.011 & 0.016 & -0.001 & -0.009 & -0.017 & -0.058 & -0.053 & 0.119 & 0.207 & 0.011 & 0.019 & 0.004 \\
\hline & ${ }^{36}(\%)$ & 0.01 & -0.03 & 0.02 & 0.06 & -0.10 & -0.02 & -0.27 & -0.08 & 0.22 & -0.02 & 0.09 & -0.11 & -0.08 \\
\hline
\end{tabular}

typical 2 uncertainties are $0.10 \%, 0.012 \%$, and $0.25 \%$ or or ${ }^{34} \mathrm{~S}$, , ${ }^{3} \mathrm{~S}$ and ${ }^{3} \mathrm{~s}_{\mathrm{S}}$ respectively

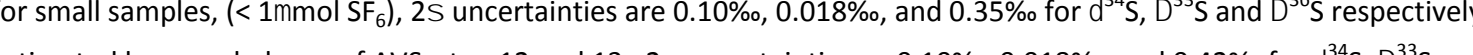

estimated by mass balance of AVS, step 12 and 13.2 uncertainties $<0.10 \%, 0.018 \%$, and $0.42 \%$ for ${ }^{34} \mathrm{~S}$, ${ }^{33} \mathrm{~S}$ and ${ }^{3{ }^{6} \mathrm{~S}}$ respectively. Typically $0.10 \%, 0.012 \%$, and $0.25 \%$,

estimated by mass balance of $\mathrm{S}^{0}$, $\mathrm{SO}_{4}$, AVS, step 12 and 13 . 2 uncertainties $<0.35 \%, 0.020 \%$, and $0.50 \%$ for ${ }^{34} \mathrm{~S}$, ${ }^{33} \mathrm{~S}$ and ${ }^{36} \mathrm{~S}$ respectively. Typically $0.30 \%, 0.012 \%$, and $0.25 \%$ 


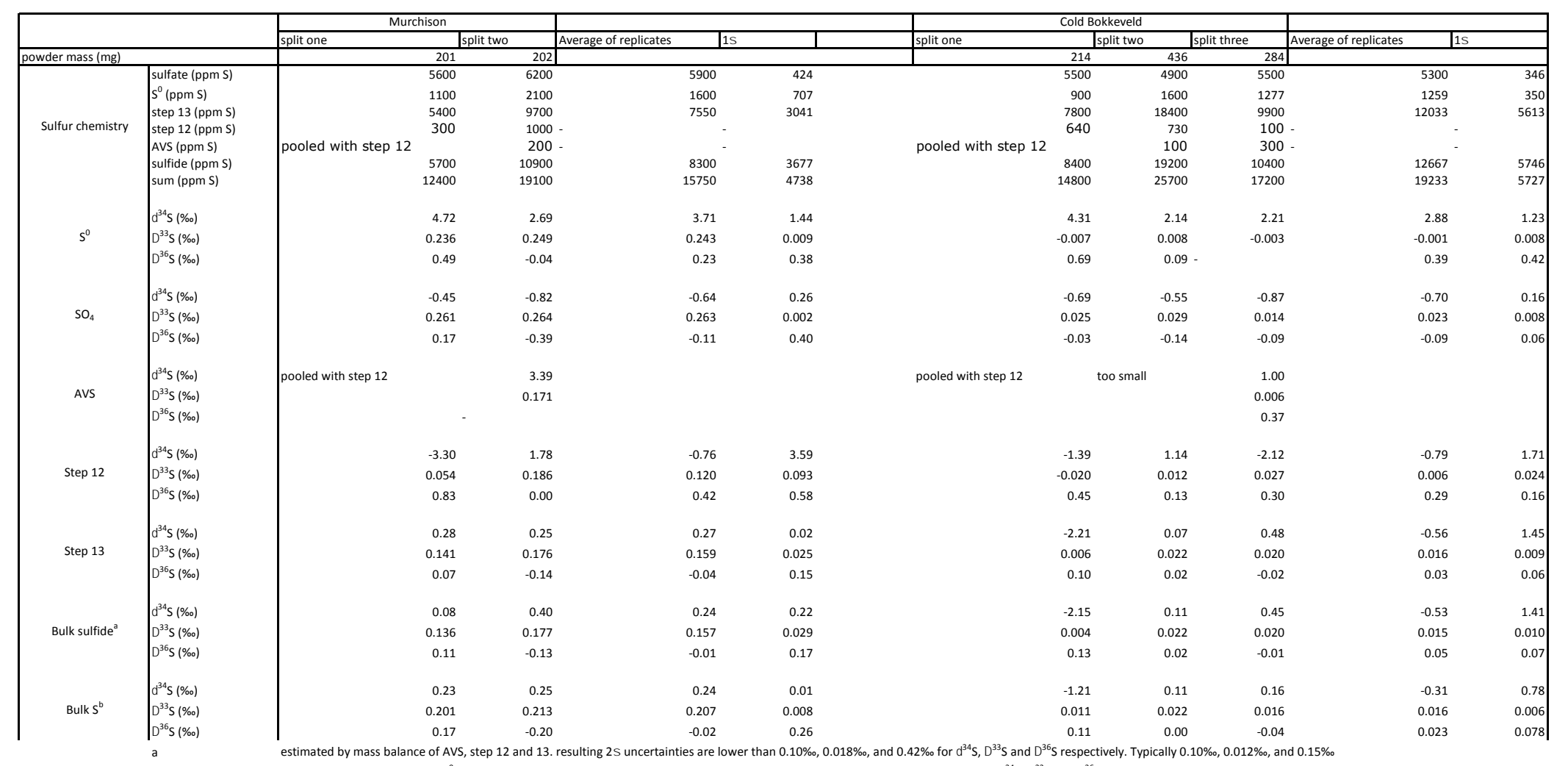

estimated by mass balance of AVS, step 12 and 13 . resulting 2 uncertainties are lower than $0.10 \%, 0.018 \%$, and $0.42 \%$ for ${ }^{34} \mathrm{~S},{ }^{33} \mathrm{~S}$ and ${ }^{36} \mathrm{~S}$ respectively. Typically $0.10 \%, 0.012 \%$, and $0.15 \%$ 
$2 \quad$ Figure 1

3 Analytical flow diagram for sulfate, $\mathrm{S}^{0}$, and sulfide extraction from CMs. Soluble and insoluble organic 4 compounds were also isolated, but are not studied here. Sulfides compositions are estimated by pooling the $5 \quad$ AVS, step 12, and step 13 extracts (see sections 3.1, 5.1.3 and appendix A).

Figure 2

8 Histograms of bulk S, sulfide, sulfate and $\mathrm{S}^{0}$ contents of the 13 CMs studied here. For all samples except $9 \quad$ PCA 91084, sulfide is the dominant pool, whereas sulfate and $S^{0}$ are minor S-carrying reservoirs.

Figure 3

12 The quadruple $\mathrm{S}$ isotopic compositions of bulk $\mathrm{S}$ in the $13 \mathrm{CMs}$ : (a) $\Delta^{33} \mathrm{~S}$ versus $\delta^{34} \mathrm{~S}$, and (b) $\Delta^{36} \mathrm{~S}$ versus

$13 \delta^{34}$ S. All the isotope data are given relative to CDT. The shaded areas are representations of CDT values and

$142 \sigma$ uncertainty. Whereas variability in $\delta^{34} S$ 国回回 $\Delta^{36} \mathrm{~S}$ remains mostly within the reported uncertainties, 15 resolvable variations are present in $\Delta^{33}$, with values varying over a $\sim 0.25 \%$ orange. Over the $13 \mathrm{CMs}, 5$ 16 samples have bulk S with non-CDT $\Delta^{33}$ S values.

\section{Figure 4}

19 For the $13 \mathrm{CMs}$, the quadruple $\mathrm{S}$ isotopic compositions of sulfide (yellow triangles), sulfate (red 20 squares) and $\mathrm{S}^{0}$ (blue circles) in the $13 \mathrm{CMs}$ : (a) $\Delta^{33} \mathrm{~S}$ versus $\delta^{34} \mathrm{~S}$, and (b) $\Delta^{36} \mathrm{~S}$ versus $\delta^{34} \mathrm{~S}$. All the isotope 21 data are given relative to CDT. Large $\Delta^{33} \mathrm{~S}$ variations are recorded in the $\mathrm{S}^{0}$ extracts. The $\Delta^{33} \mathrm{~S}$ values vary 22 over a $\sim 0.35 \%$ range. This is also observed in the sulfate extracts, but the sulfide extracts mimic the bulk S 23 compositions with moderate $\Delta^{33} \mathrm{~S}$ variations that range over $\sim 0.2 \%$. In contrast to bulk S, resolvable $24 \delta^{34} \mathrm{~S}$ 国回回 $\Delta^{36} \mathrm{~S}$ are present in the individual $\mathrm{S}$ components. $\mathrm{A} \sim 10 \%$ o $\delta^{34} \mathrm{~S}$ range is observed between 25 sulfate and $\mathrm{S}^{0}$. The $\Delta^{36} \mathrm{~S}$ values vary over a $\sim 1 \%$ range.

\section{Figure 5}




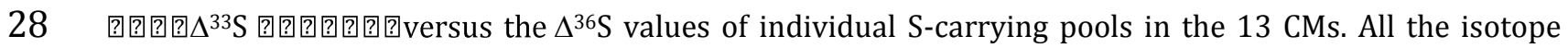

29 data are given relative to CDT. The samples show a rough negative trend, with a slope of $-3.9 \pm 2.2(2 \sigma)$. The

30 slope is better defined if only $S^{0}$ is considered, with a value of $-3.1 \pm 1.0(2 \sigma)$. Murchison and MCY 05230 are

31 excluded from this main trend, both showing positive $\Delta^{36} \mathrm{~S} / \Delta^{33} \mathrm{~S}$ values for their sulfide, sulfate and $\mathrm{S}^{0}$. The

32 main trend and the deviations from the main trend (for Murchison and MCY 05230) provide information

33 on the type of $\mathrm{H}_{2} \mathrm{~S}$ photodissociation that occurred in the nebula (see section 5.4). plotted against (a) the sulfate, and (b) the sulfide values. The 1:1 slopes are shown in red. The $\mathrm{S}^{0}$-sulfate trend is indistinguishable from a 1:1 slope (MCY 05230 is excluded). For all CMs except MCY 05230, this suggests a direct link between sulfate and $S^{0}$ (see section 5.2). Sulfide and $S^{0}$ may be linked through their condensation sequence or via the delivery of late sulfide to the CMs that partially oxidize to $\mathrm{S}^{0}$ (see section

\section{Figure 6}

An evaluation of the genetic links between the S-carrying phases in CMs. The $\Delta^{33} \mathrm{~S}$ values of $\mathrm{S}^{0}$ are trend is indistinguishable from a $1: 1$ slope (MCY 05230 is excluded). For all CMs except MCY 05230, this 5.4)

\section{Figure 7}

The $\Delta^{33} \mathrm{~S}$ values of $\mathrm{S}^{0}$, sulfate and sulfide, in 12 CMs (PCA 91084, the only heated CM, is excluded). The isotope data are given relative to CDT. Chondrites are ranked by order of increasing $\Delta^{33} \mathrm{~S}$ values in $\mathrm{S}^{0}$. The values for $\mathrm{S}^{0}$ are compared with (a) sulfate and (b) sulfide.

\section{Figure 8}

50 Schematic representation of the $\mathrm{H}_{2} \mathrm{~S}$ photodissociation under open-system Rayleigh distillation 51 conditions as described in model A (see section 5.4.1.). The $\mathrm{x}$-axis is the remaining fraction of $\mathrm{H}_{2} \mathrm{~S}$ in the 52 nebula. The y-axis is $\Delta^{33} \mathrm{~S}$, shown for the remaining $\mathrm{H}_{2} \mathrm{~S}$ and the instantaneous product $\mathrm{S}^{0}$. The star symbols 53 are used for illustrating the composition of $\mathrm{H}_{2} \mathrm{~S}$. The composition of the initial $\mathrm{H}_{2} \mathrm{~S}$ reservoir and $\mathrm{H}_{2} \mathrm{~S}$ likely 54 recorded by Murchison and MCY 05230 sulfide pools are shown. The hexagon symbol is used for 
55 illustrating the composition of produced S0 for GRA 98074 as an example (section 5.4.1). For the first and

56 second photodissociation regime $(f>0.2$ and $f<0.2$, respectively $), \Delta^{33}$ S fractionations are taken at $-0.1 \% 0$

57 and $+0.3 \%$, respectively. For most CMs, $\Delta^{33} \mathrm{~S}$ values of $\mathrm{S}^{0}$ are lower than for sulfide, as accounted for by the

58 first photodissociation regime. Additionally, it corresponds to $\Delta^{36} \mathrm{~S} / \Delta^{33} \mathrm{~S}$ values for $\mathrm{CMs}$ at $-3.1 \pm 1.0$,

59 consistent with $\mathrm{H}_{2} \mathrm{~S}$ photodissociation occurring at $\lambda$ between 120 and $139 \mathrm{~nm}$. The one example of GRA

6098074 is taken for illustration here. The conclusion does not change if other CMs were taken for illustration

61 (section 5.4.1). For Murchison and MCY 05230, the $\Delta^{33} S$ values of $S^{0}$ are higher than for sulfide, and the

$62 \Delta^{36} \mathrm{~S} / \Delta^{33} \mathrm{~S}$ values are positive, indicating a change in the main wavelength responsible for the

63 photodissociation (sections 5.4.1. and 5.4.2.). The minimum $\Delta^{33} \mathrm{~S}$ value of $\mathrm{H}_{2} \mathrm{~S}$ at the transition between the

64 two regimes requires a low $f$ value (here 0.2 ) as constrained by the Murchison value of $+0.156 \pm 0.008 \%$.

65 This requires substantial processing of $\mathrm{H}_{2} \mathrm{~S}$ for its isotopic composition to have evolved significantly from

66 CDT. We suggest here that the photodissociation process recorded by Murchison and MCY 05230 could

67 postdate the recorded in the other chondrites studied here. Note that with a $\Delta^{36} S / \Delta^{33} S$ of $\sim-3$, the first

68 photodissociation regime would lead the $\Delta^{36}$ S values for Murchison and MCY 05230 to be $-0.45 \pm 0.30 \%$,

69 which is not seen (these CMs have $\Delta^{36}$ S values closer to zero, Table 3). Although the large uncertainties on

$70 \Delta^{36} \mathrm{~S}$ values for these two CMs do not allow invalidating the present prediction, a preferred alternative

71 model is developed in section 5.4.3.

72

73

\section{Figure 9}

74 The $\mathrm{S}$ isotopic relation between sulfate and its potential precursors: $\mathrm{S}^{0}$ and sulfide. (a) The $\delta^{34} \mathrm{~S}$

75 difference between sulfate and $S^{0}$ is plotted versus their $\Delta^{33} S$ difference. (b) The difference between sulfate

76 and sulfide. The occurrence of at least three outliers in the trend between sulfate and sulfide argues against

77 a direct genetic link between sulfate and sulfide. There is a direct negative trend between $\mathrm{S}^{0}$ and sulfate.

78 This suggests a sulfate origin through $\mathrm{S}^{0}$ oxidation in the CM parent body (see also fig. 6). The ${ }^{34}$ S depletion

79 in sulfate, relative to $\mathrm{S}^{0}$, indicates that the oxidation process is a normal kinetic mechanism. The more

80 sulfates are depleted in ${ }^{34} S$ relative to $S^{0}$, the higher their $\Delta^{33} S$ are relative to $S^{0}$. We estimate that the

$81{ }^{33} \mathrm{~S} /{ }^{32} \mathrm{~S}$ fractionation is $0.505 \pm 0.005$ times that of the ${ }^{34} \mathrm{~S} /{ }^{32} \mathrm{~S}$ fractionation. As observed for the first time in 
82 natural samples, this relationship indicates that the kinetic oxidation process is characterized by a non83 canonical mass dependency (see details in section 5.2).

84

85 


\section{Powdered sample}

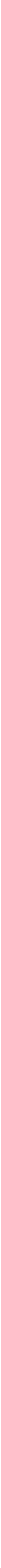



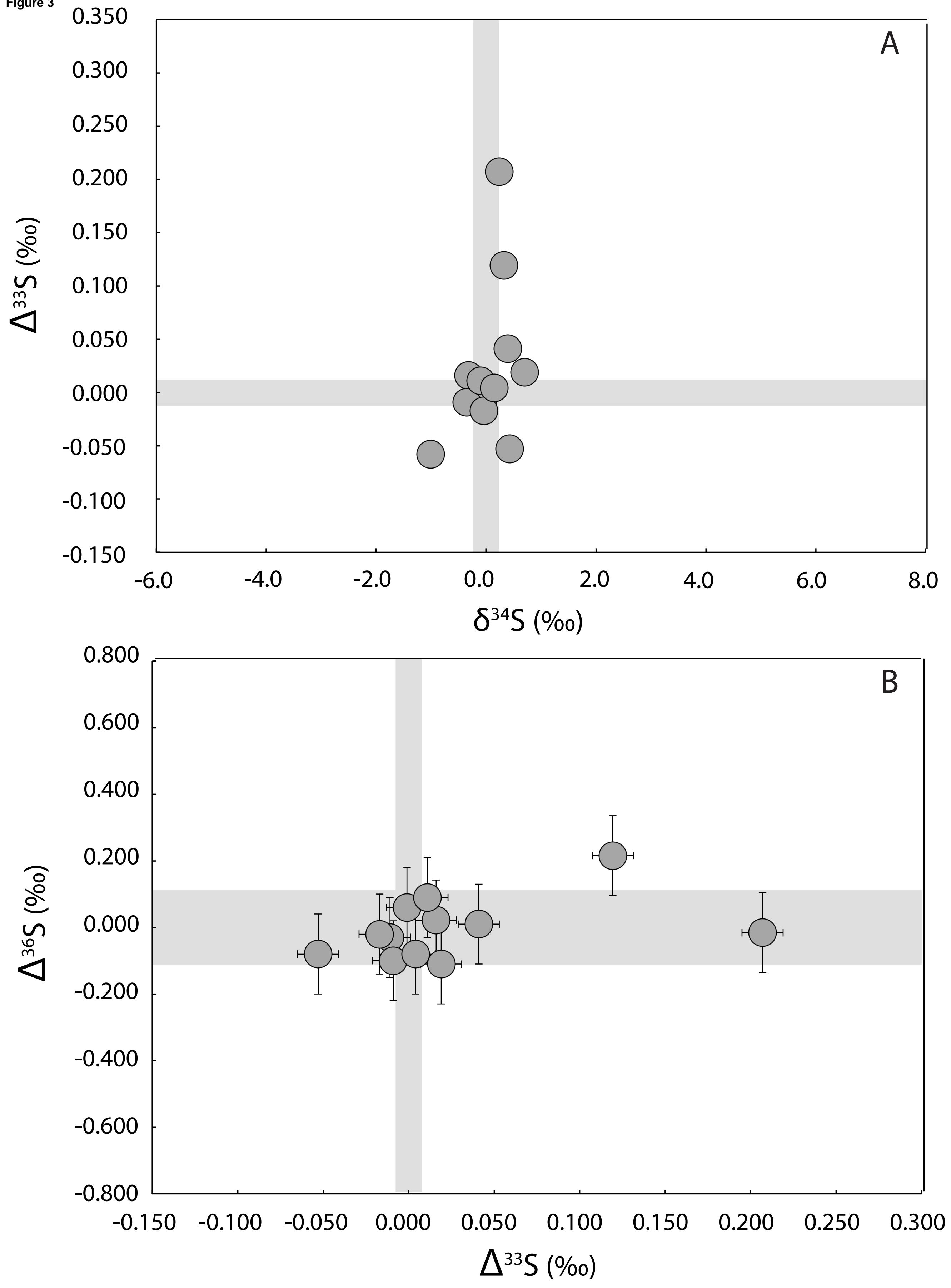

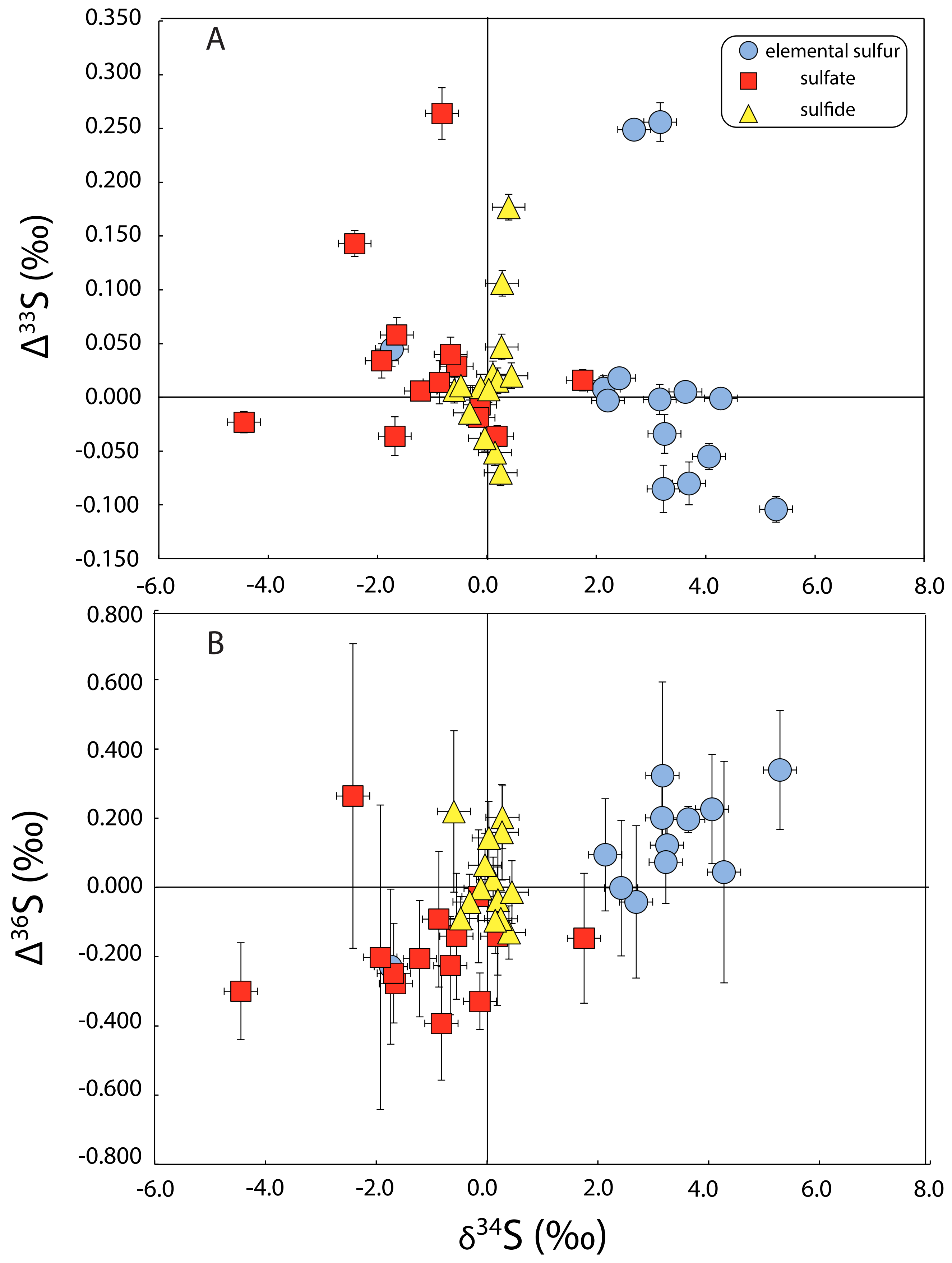


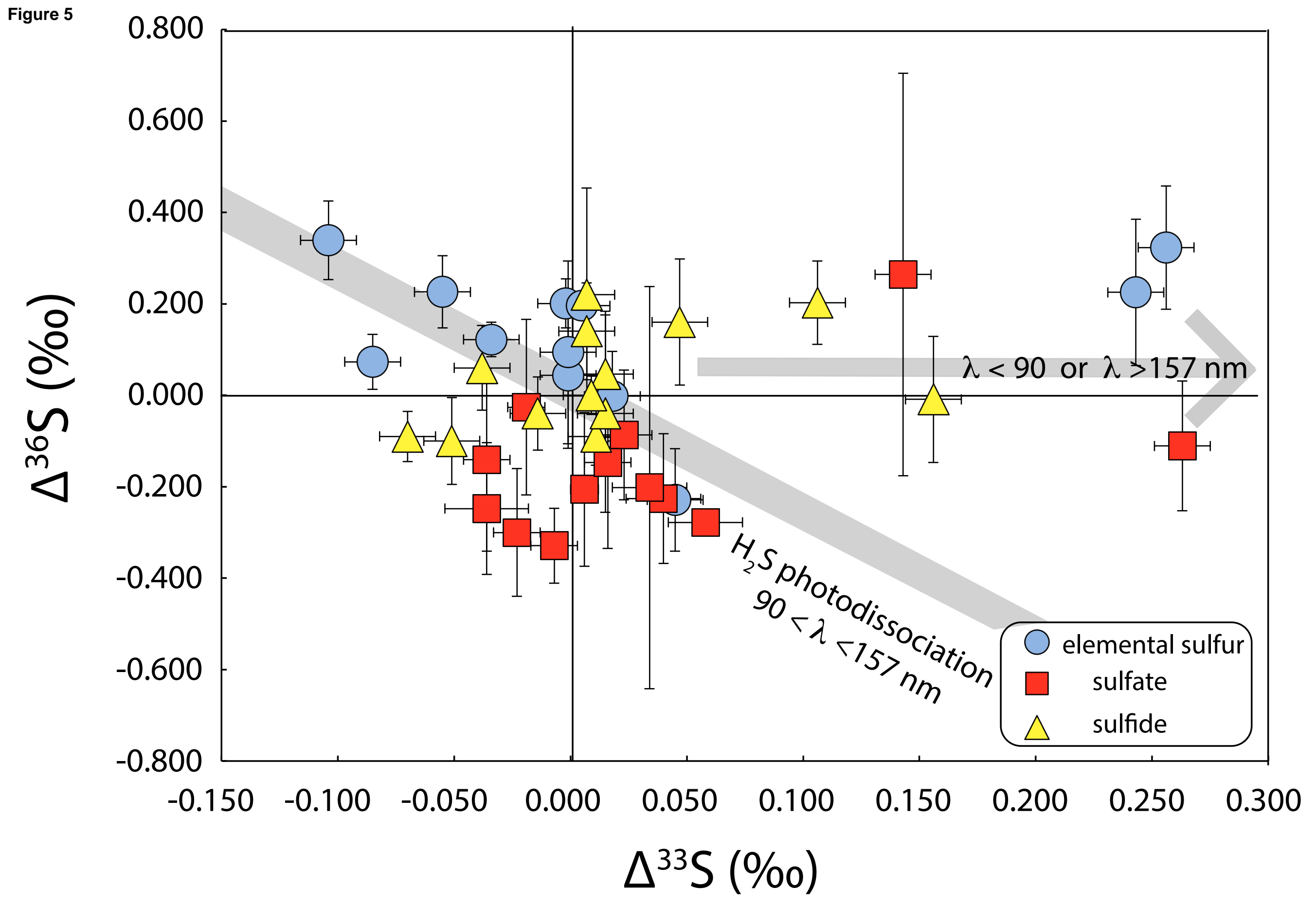




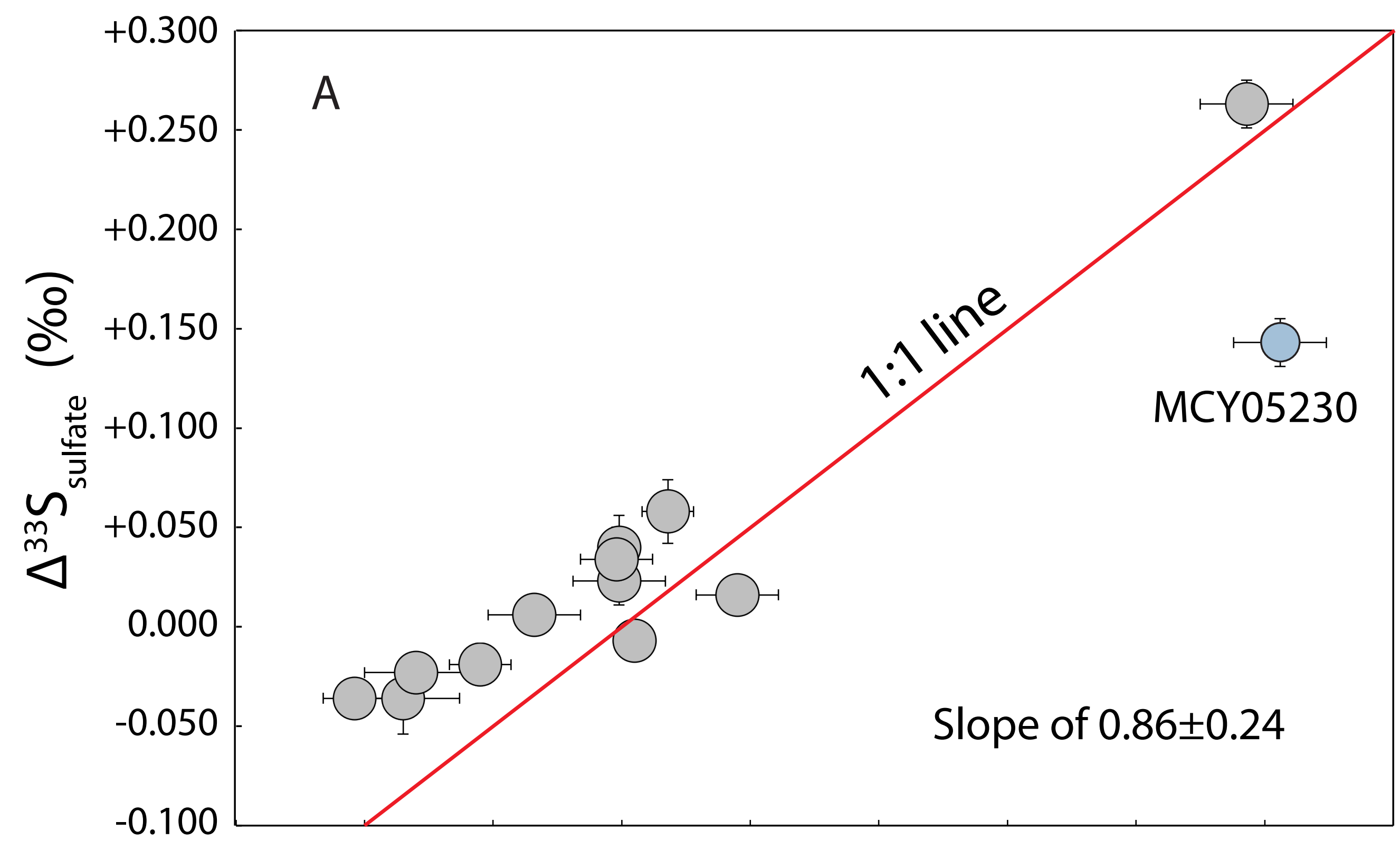

$-0.150-0.100-0.050 \quad 0.000+0.050+0.100+0.150+0.200+0.250+0.300$

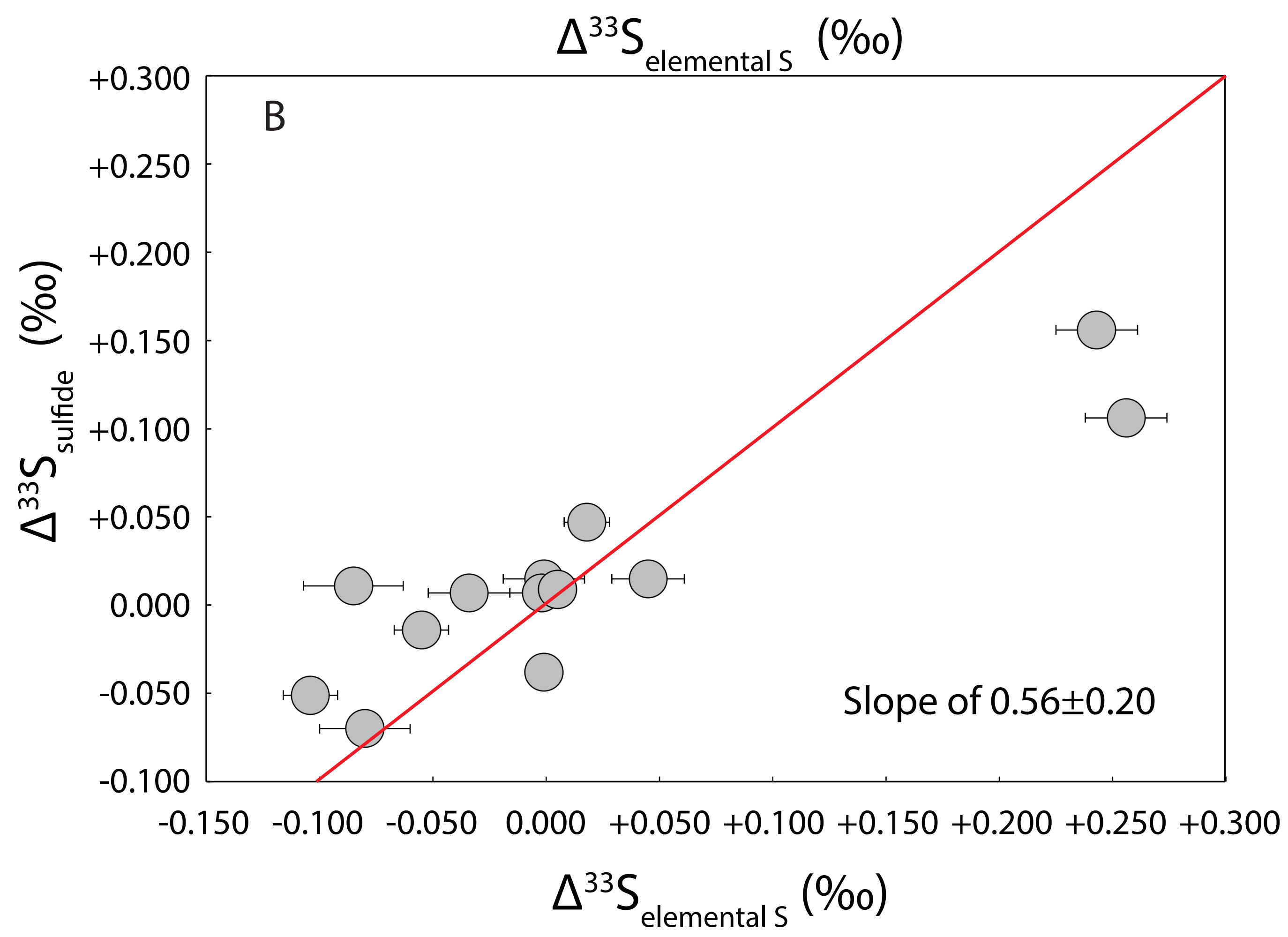




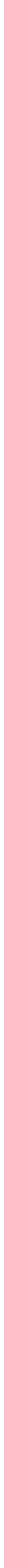




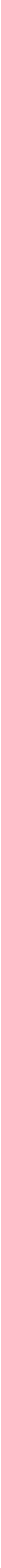

Figure 9 

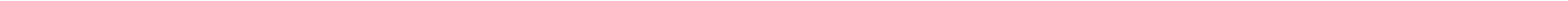
